\title{
Dosis de metadona y su relación con calidad de vida, satisfacción, psicopatología, rendimiento cognitivo y consumo adicional de sustancias no prescritas
}

\author{
Methadone dosage and its relationship to quality of life, \\ satisfaction, psychopathology, cognitive performance \\ and additional consumption of non-prescribed drugs
}

\author{
Eduardo J. Pedrero-Pérez*, Grupo MethaQoL** \\ * Instituto de Adicciones. Madrid Salud; ** Instituto de Adicciones. Madrid Salud y Junta de Extremadura \\ (listado completo al final).
}

\section{Resumen}

La efectividad de los tratamientos con metadona está fuera de toda duda, si bien persisten dudas sobre las dosis efectivas y los objetivos que debe perseguir un programa de mantenimiento. Algunos autores propugnan que sólo superiores a 50-60 mg/día deben ser consideradas efectivas, al bloquear los receptores opioides. Otros proponen dosis ajustadas a las necesidades del paciente, atendiendo prioritariamente a su recuperación. Se estudió una muestra representativa de todos los pacientes en tratamiento con agonistas del Instituto de Adicciones de Madrid ( $\mathrm{N}=1898, \mathrm{n}=450)$ y de la Junta de Extremadura $(\mathrm{N}=100$, $\mathrm{n}=65$ ). Se evaluaron calidad de vida, satisfacción con el tratamiento, sintomatología psicopatológica, rendimiento cognitivo y consumos adicionales. Los resultados muestran una relación negativa entre dosis y calidad de vida, sintomatología psicopatológica y rendimiento cognitivo. La satisfacción con el tratamiento, basado en dosis negociadas entre médico y paciente, fue muy elevada, con independencia de la dosis. Se formuló una ecuación estructural que relacionara todas las variables. Los resultados descartan la necesidad de utilizar dosis altas si el paciente no las precisa, y contar con otras intervenciones psicosociales que favorezcan la recuperación frente a la cronificación que supone el uso de dosis altas. Mientras los programas de altas dosis atienden prioritariamente a indicadores de control social, la calidad de vida del paciente debe ser uno de los principales indicadores de éxito del tratamiento, como en cualquier otro problema de salud.

Palabras clave: Mantenimiento con metadona; Dosis; Calidad de vida; Adicción; Tratamiento.

\begin{abstract}
The effectiveness of methadone maintenance treatment is beyond any doubt, but there remains some incertitude about the appropriate and effective dosage and the objectives that should be achieved by this therapy. Some authors maintain that only doses higher than $50-60 \mathrm{mg} /$ day ought to be considered effective, since only these block all the opioid receptors. But others propose the use of doses adjusted to the needs of the patient, based on their recovery process. Quality of life, satisfaction with treatment, psychopathological symptoms, cognitive performance and additional intake of illegal and unprescribed drugs were evaluated in a representative sample of all patients treated with opioid agonists in the Addiction Institute of Madrid $(\mathrm{N}=1898, \mathrm{n}=450)$ and the Junta de Extremadura $(\mathrm{N}=$ $100, \mathrm{n}=65)$. The results revealed a negative relationship between dose and quality of life, psychopathological symptoms and cognitive performance. Satisfaction with treatment, based on doses negotiated together by doctor and patient, was very high, regardless of the dose. To establish hypothetical causal dependencies among the studied variables structural equation modelling was performed. The results reject the need for high dosage if not required by the patient, and highlight the benefits of other psychosocial interventions that lead to recovery, despite the chronification that could imply the use of high doses. Whereas high dosage programmes provide better indicators of social control, the patient's quality of life must be one of the main indicators of a successful treatment, as in any other health problem. Keywords: Methadone Maintenance; Dosage; Quality of Life; Addiction; Treatment.
\end{abstract}




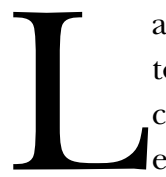

a eficacia, efectividad y eficiencia de los tratamientos con metadona en el tratamiento de personas con adicción a heroína está fuera de toda duda en el momento actual (Mattick, Breen, Kimber y Davoli, 2009). Sin embargo, persisten dudas sobre cuáles son las dosis más efectivas y cuáles los objetivos que debe perseguir un programa de mantenimiento.

La línea predominante es la que aboga por la prescripción de dosis superiores a 50-60 mg/día (90-100 mg como media), considerando que un tratamiento de sustitución debe perseguir tres objetivos prioritarios: (a) supresión de los síntomas de la retirada de opiáceos exógenos; (b) extinción del craving; y (c) bloqueo farmacológico de la capacidad reforzante de la heroína por saturación de los receptores opioides (Maremmani, Pacini, Lubrano y Lovrecic, 2003). Los principales indicadores de éxito de tratamiento serían la reducción en el consumo de heroína y cocaína, la reducción de la gravedad de los problemas relacionados con el consumo y mayores tasas de retención. Este enfoque atiende primordialmente a los efectos farmacológicos de los opiáceos y su capacidad de bloqueo de los receptores (Pacini, Maremmani, Rovai, Rugani y Maremmani, 2010). Diversos estudios (p. ej., Adelson et al., 2013; Faggiano, Vigna-Taglianti, Versino y Lemma, 2003; Farré, Mas, Torrens, Moreno y Camí, 2002) encuentran que dosis más altas se corresponden con más tiempo en tratamiento y con menos consumos de heroína y otras drogas. Los pacientes con psicopatología asociada requieren dosis mayores, $150 \mathrm{mg}$ / día, que quienes sólo presentan adicción a opiáceos, que requerirían como media $100 \mathrm{mg}$ /día (Eiden, Leglis, Clarivet, Blayac y Peyrière, 2012). Otros autores abogan incluso por dosis muy altas (desde 100 hasta $780 \mathrm{mg}$ /día) como "necesarias" para evitar el consumo de opiáceos y controlar la psicopatología coexistente (Maxwell y Shinderman, 1999).

Este enfoque ha recibido críticas importantes, en la medida en que desatiende otras cuestiones, como la perspectiva del propio paciente o la necesidad de atender prioritariamente otros problemas y riesgos. Es necesario tener en cuenta que, como sucede con el resto de opiáceos (Katz, 2005; Benyamin et al., 2008), la metadona no es un fármaco exento de efectos secundarios e indeseados (Bell y Zador, 2000; Bileviciute-Ljungar, Häglund, Carlsson y von Heijne, 2014; Chugh et al., 2008; Grönbladh y Öhlund, 2011; Webster, 2013), cuya probabilidad de aparición e intensidad se incrementan en paralelo a las dosis (Leavitt, 2003; Walker, Klein y Kasza, 2003), apareciendo con cierta frecuencia complicaciones de gran envergadura a dosis altas (Krantz, Kutinsky, Robertson y Mehler, 2003), aunque también a dosis no tan altas (Krantz, Martin, Stimmel, Mehta y Haigney, 2009; Roy et al., 2012). Entre estos efectos secundarios, los déficits en el rendimiento neuropsicológico están entre los más frecuentemente identificados (Bracken et al., 2012; Gruber et al., 2006; Loeber, Kniest, Diehl, Mann y Croissant, 2008; Mintzer, Copersino y Stitzer, 2005; Mintzer y Stitzer, 2002; Rass et al., 2014) y se incrementan en paralelo a las dosis (Rass et al., 2014). Los pacientes en tratamiento con metadona presentaron déficits cognitivos significativos, mientras que quienes estaban en abstinencia prolongada de opiáceos y sin tratamiento rendían significativamente mejor (Verdejo, Toribio, Orozco, Puente y Pérez-García, 2005), incluso como los controles (Darke, McDonald, Kaye y Torok, 2012).

Desde otra perspectiva, se han propuesto los denominados programas de "bajas dosis" (o dosis subumbral: low thresold programs), cuyo objetivo principal no es necesariamente eliminar el uso de drogas ilícitas, sino establecer y mantener contacto con los consumidores de opiáceos, con el fin de ayudar a estabilizar y reducir algunos de los riesgos asociados, y desarrollar la confianza necesaria para aspirar a objetivos más ambiciosos en fases posteriores (Hartgers, van den Hoek, Krijnen y Coutinho, 1992). Existe abundante apoyo empírico que ratifica la mejora sustancial de la calidad de vida de estos pacientes y la reducción del riesgo de complicaciones de gran envergadura, a pesar de que no se produzca una interrupción completa del consumo (p. ej., Brugal et al., 2005; Millson et al., 2007; Torrens, Castillo y Perez-Sola, 1996; Villeneuve et al., 2006). Algunos estudios muestran que la retención en este tipo de programas no es inferior a la de otros que utilizan dosis más elevadas (Perreault et al., 2007) y que pueden favorecer la incorporación a otras modalidades de tratamiento cuando se requiere (Schwartz et al., 2006).

Una tercera línea de tratamientos se caracteriza por focalizar su evaluación en la mejora de la calidad de vida, sin prestar atención especial a la dosis requerida para ello. Cuando se tiene en cuenta la calidad de vida de los pacientes es preciso considerar también la necesidad de llevar a cabo intervenciones de índole psicosocial para evitar las consecuencias negativas de los tratamientos: estigmatización, discriminación, dependencia de la metadona y los efectos paralizantes de la droga en las emociones (De Maeyer, Vanderplasschen, Camfield et al., 2011; Harris y McElrath, 2012). El éxito del tratamiento depende de otros factores, tales como el empleo, las relaciones familiares, la disponibilidad de relaciones íntimas, la estructuración horaria de las actividades de la vida diaria y el cambio de hábitos relacionados con la salud, entre otros (De Maeyer, Vanderplasschen, Lammertyn et al., 2011; He et al., 2011). Estos programas parten de la consideración de que la respuesta al tratamiento es función de diferencias individuales y no una mera función farmacológica dosis-respuesta (Padaiga, Subata y Vanagas, 2007). Desde esta perspectiva, no cabe hablar de dosis altas o bajas, sino dosis ajustadas o adecuadas, que eliminen la necesidad (pero no la posibilidad) de que se produzcan consumos adicionales. En términos generales, suele encontrarse que el tratamiento de mantenimiento con metadona se traduce en un inmediato incremento en la calidad de vida, que se suaviza con el paso del tiempo, de modo que no llega a alcanzar el encontrado en población general, ni siquiera al que declaran sujetos con otros trastornos psi- 
copatológicos graves (Habrat, Chmielewska, Baran-Furga, Keszycka y Taracha, 2002; Karow et al., 2011; Millson et al., 2004; Nosyk, Marsh, Sun, Schechter y Anis, 2010; Nosyk et al., 2011; Torrens, Domingo-Salvany, Alonso, Castillo y San, 1999) y que las variables más relacionadas con la calidad de vida y el éxito del programa no son las relacionadas con la sustancia, sino con factores psicosociales, como el apoyo familiar (Lina, Wu y Detels, 2011). La multiplicidad de factores implicados en la gravedad de la adicción y la calidad de vida autopercibida por los pacientes indica la necesidad de diseñar programas que atiendan a las múltiples dimensiones implicadas en el problema (Fernández Miranda, González García-Portilla, Sáiz Martínez, Gutiérrez Cienfuegos y Bobes García, 1999; Millson, et al., 2006). Y, sin embargo, la calidad de vida no es uno de los indicadores de resultados que se utilizan para medir la efectividad de los tratamientos (Amato et al., 2005; Fernández Miranda, 2000).

El hallazgo repetido de que las dosis altas incrementan la retención ha sido desafiado por algunos autores, que encuentran que el riesgo de abandono es mayor (1,3/1) cuando las dosis superan los $60 \mathrm{mg} /$ día, que cuando no alcanzan esta dosis, siendo otros los factores que predicen el éxito o fracaso de los programas de mantenimiento (Mino, Page, Dumont y Broers, 1998). En cuanto al consumo de drogas no prescritas, también hay estudios que cuestionan la superioridad de los programas de altas dosis, considerando que una adecuada intervención psicosocial complementaria a dosis bajas puede obtener iguales o mejores resultados que las dosis altas (Banys, Tusel, Sees, Reilly y Delucchi, 1994). Contra lo propuesto por el modelo de altas dosis, otros estudios encuentran que el incremento de dosis de metadona por encima de los niveles de ajuste provoca un incremento notable del craving y del consumo de heroína (Curran, Bolton, Wanigaratne y Smyth, 1999; Fareed et al., 2010). Estudios de seguimiento en Estados Unidos han mostrado que la dosis mínima de metadona sugerida por el modelo de altas dosis (60 mg) no es considerada como necesaria en la clínica, y que la tendencia entre los médicos prescriptores es hacia prestar cada vez más atención a la opinión de los pacientes para establecer la dosis adecuada que a las políticas de dosificación (D’Aunno, Folz-Murphy y Lin, 1999), de modo que tener en cuenta la opinión del paciente en el establecimiento de las dosis mejora los resultados (Maddux, Desmond y Vogtsberger, 1995; Maddux, Prihoda y Vogtsberger, 1997). Un estudio realizado en España, con una muestra representativa de todo el territorio nacional, encontró que la dosis media de mantenimiento era de $61,52 \mathrm{mg} /$ día (d.t. $=49,14$ ), lo que significa que un alto porcentaje de los pacientes recibirían dosis inferiores a $60 \mathrm{mg}$ /día (Roncero et al., 2011). Otros autores encuentran irrelevante la dosis en la consecución de objetivos, sugiriendo que debe prestarse mayor atención a otros aspectos del programa, como la relación interpersonal entre terapeuta-paciente (Blaney y Craig, 1999). Sin embargo, todos los estudios que exploran variables relacionadas con dosis menores de $90 \mathrm{mg} /$ día han ido desapareciendo de la literatura, en la medida en que las guías recomiendan, de modo insistente, la prescripción de dosis altas (D’Aunno, Pollack, Frimpong y Wuchiett, 2014).

Pocos estudios se han centrado en conocer la opinión de los pacientes, su percepción de salud en relación a las dosis y la influencia de sus actitudes y otras variables psicológicas en relación a los resultados del tratamiento. Diversos estudios informan de amplias discrepancias entre la estimación de resultados declarados por los profesionales y los que son percibidos por los pacientes (Trujols et al., 2013). Si bien la motivación es una variable decisiva para la obtención de buenos resultados en el tratamiento, con independencia de las dosis administradas (Zeldman, Ryan y Fiscella, 2004), muchos estudios coinciden en constatar que los pacientes encuentran diversas barreras para entrar y permanecer en los programas de metadona: el trato recibido por el equipo terapéutico y la consideración de "enfermos", los largos tiempos de espera, la inflexibilidad en la prescripción de dosis, la ocultación de las dosis recibidas, la probable estancia indefinida, la consideración de recibir dosis demasiado altas o la falta de participación requerida en su dosificación, entre otros (p. ej., Al-Tayyib y Koester, 2011; Deering et al., 2011; Peterson et al., 2010). En sentido contrario, la satisfacción con el tratamiento recibido, la asistencia a actividades psicoterapéuticas y la sensación de que el tratamiento resulta beneficioso, son cuestiones que mejoran la retención con independencia de las dosis recibidas (Kelly, O'Grady, Brown, Mitchell y Schwartz, 2010; Montgomery, Sanning, Litvak y Peters, 2014; Vanderplasschen, Naert, Vander Laenen y De Maeyer, 2014), de modo que la satisfacción presenta una capacidad predictiva sobre la retención de la que carece la dosis administrada (Kelly, O'Grady, Brown, Mitchell y Schwartz, 2011). La mejora de la calidad de los tratamientos con metadona, como tratamientos biopsicosociales de efectividad contrastada, pero adaptables a los diferentes perfiles y necesidades, es un objetivo irrenunciable, como lo es la propia opinión de los pacientes (Fernández Miranda, 2004; Rodríguez et al., 2002).

El objetivo del presente trabajo es encontrar evidencia empírica que aconseje el uso de dosis altas cuando se tiene en consideración la perspectiva del paciente. Para ello, se someterán a contrastación algunas hipótesis derivadas de los estudios revisados: (a) que las dosis altas se corresponden con mayores niveles de calidad de vida autopercibida; (b) que la prescripción de dosis altas se corresponde con una mayor satisfacción con el tratamiento; (c) que las personas que reciben dosis altas manifiestan menores niveles de malestar somático y psicológico; (d) que las dosis altas se corresponden con rendimiento cognitivo igual o superior al de las dosis bajas; (e) que los sujetos que reciben dosis altas presentan menos consumos de drogas no prescritas que aquellos que reciben dosis bajas. Adicionalmente, se pretende encontrar la relación que todas estas variables muestran 
sobre la dosis recibida en un modelo estructural que sugiera una hipótesis causal.

\section{Método}

\section{Descripción de los dispositivos}

El estudio se ha realizado sobre dos muestras, ambas obtenidas en dispositivos públicos específicos: una muestra principal, obtenida en una gran ciudad (Madrid) y otra obtenida en ciudades pequeñas que atienden a población urbana y rural (Extremadura). El Instituto de Adicciones es un organismo público dependiente del Ayuntamiento de Madrid, que proporciona atención a personas con problemas relacionados con el consumo de drogas u otros comportamientos adictivos sin sustancia de todo el municipio de Madrid (población aproximada 3.200.000 habitantes). El territorio de la ciudad está dividido en 7 sectores, correspondiendo la población de cada uno de ellos a un Centro de Atención a Drogodependientes (CAD), de gestión pública directa. Además, existen otros 3 centros de tratamiento concertados con organizaciones no gubernamentales (Cáritas y Cruz Roja), con financiación pública y gestión indirecta (CCAD). Los 10 servicios participantes cuentan con equipos de tratamiento multidisciplinares (médicos, enfermeros, psicólogos, trabajadores sociales, terapeutas ocupacionales y personal auxiliar). El acceso de los pacientes a los servicios puede ser directo, a iniciativa propia, o mediante la derivación desde otros servicios sanitarios: médico de familia, salud mental u hospitales. El tratamiento que se proporciona es individualizado, atendiendo a las necesidades médicas, psicológicas, ocupacionales y sociales de cada paciente. En personas con consumo activo de heroína, puede priorizarse la evaluación médica e iniciar el tratamiento con metadona de inmediato, demorando el resto de evaluaciones de las demás áreas. Dentro de cada servicio, existe un Programa de Tratamiento con Agonistas Opiáceos, en el que participan todos los profesionales. La prescripción de metadona o buprenorfina corresponde a los médicos, que no tienen instrucciones estrictas sobre normas de dosificación, de modo que pueden prescribir en función de su relación con el paciente y de sus propios criterios. Los pacientes mantienen citas individuales periódicas con sus médicos (y con el resto de profesionales), pudiendo manifestar su sintomatología y su deseo de subirlas o bajarlas, siendo el profesional médico quien, en último término, prescribe la dosis que considere más adecuada a las características y situación del paciente. La sustancia administrada es, en todo caso, clorhidrato de metadona (aunque existe un subprograma de buprenorfina, no incluido en el presente estudio), en disolución o en comprimidos, y su recogida en el centro es diaria, dos veces en semana o una vez semanal (dosis take home).

La muestra de comparación (a la que denominaremos muestra B) se obtuvo de diversos centros de tratamiento ambulatorio de Extremadura. La dinámica de estos centros es similar a la expuesta para Madrid, pero la población que atiende es sensiblemente diferente: ciudades pequeñas (Cáceres alrededor de 95.000 habitantes, Badajoz 150.000) y zonas rurales aledañas.

\section{Participantes}

$\mathrm{Al}$ inicio del estudio, se encontraban en tratamiento en todos los centros de la red del Instituto de Adicciones Madrid un total de 1898 pacientes, distribuidos en 10 centros. Todos estos centros contaban con financiación pública y eran de carácter gratuito, atendiendo a pacientes del municipio de Madrid. Para el presente estudio, se estableció un intervalo de confianza máximo del $4 \%$ ( $\mathrm{p}=\mathrm{q}=0,5)$, lo que suponía la obtención de un $\mathrm{n}=450$ sujetos. Las evaluaciones se realizaron entre enero de 2014 y enero de 2015, realizándose un total de $n=538$ evaluaciones, aunque tras la anulación de 80 evaluaciones por errores en la cumplimentación de las pruebas o ausencia de datos, la muestra final (muestra principal) quedó establecida en $n=458$ sujetos. Como criterio de inclusión se estableció el hecho de recibir metadona prescrita por dependencia de heroína durante al menos 3 meses en el servicio correspondiente. Como criterios de exclusión se determinaron: diagnóstico de dependencia de alguna sustancia diferente a la heroína, consumo reciente de alcohol, padecer algún tipo de daño cerebral, sintomatología psicótica aguda, recibir tratamiento farmacológico (antirretroviral o de otro tipo) que obligara a la modificación de dosis de metadona, dificultades para la comprensión del idioma español o cualquier otra que comprometiera la adecuada resolución de las pruebas. La muestra B se obtuvo de los diferentes centros públicos de tratamiento de Extremadura. La población total en tratamiento al inicio del estudio era de 100 sujetos, obteniéndose evaluaciones de las $2 / 3$ partes $(n=65)$. A pesar de ello, la muestra resultó representativa, pero con un elevado error (intervalo de confianza $7 \%$ para $p=q=0,5$ ).

\section{Instrumentos}

Cuestionario de Calidad de Vida de la Organización Mundial de la Salud, versión abreviada (World Health Organization Quality of Life, WHOQOL BREF; WHO, 2004), instrumento desarrollado con intención de contar con una evaluación de calidad de vida aplicable en todas las culturas. La versión completa consta de 100 ítems, y la abreviada -utilizada en el presente trabajo- de 26: 2 preguntas generales (sobre calidad de vida general y satisfacción con la salud) y 24 ítems que abarcan cuatro dominios: salud física, psicológica, social y ambiental. Los ítems se responden en una escala tipo Likert de 5 opciones. Se han estudiado sus propiedades psicométricas en estudios transculturales (Skevington, Lotfy y O’Connell, 2004) y en población española (Lucas-Carrasco, 2012). Se ha utilizado la versión proporcionada por el Servicio Andaluz de Salud (2010). La consistencia interna de la prueba en la muestra de estudio fue de $\alpha=0,89$, con una correlación ítem test corregida $0,30<$ rit $<0,63$. 
Escala de Satisfacción con el Tratamiento de Metadona, elaborada a partir de la Verona Service Satisfaction Scale de 32 ítems (VSSS-32; Ruggeri et al., 2000), validada en población clínica española (Trujols y Pérez de los Cobos, 2005), pero modificada para adaptarla a las características de los servicios participantes (Anexo I). Consta de 13 ítems que se responden en una escala tipo Likert de 5 opciones, en la que se valoran los aspectos del tratamiento en términos generales, y 8 ítems en los que se pregunta si ha recibido un tipo de atención determinada y, en caso de respuesta afirmativa, se procede a una valoración similar. Para la puntuación general de Satisfacción con el Tratamiento se consideraron las puntuaciones de los 13 primeros ítems, multiplicando el resultado por 25 para obtener una puntuación entre 0 y 100 puntos, con una media de 50. Las consistencia interna de la prueba fue satisfactoria, con un $\alpha=0,86$ para los 29 ítems y $\alpha=0,91$ para los 13 primeros ítems, con una correlación ítem test corregida $0,70<$ rit $<0,88$.

Inventario de 90 Síntomas Revisado (Symtomps Check List 90 Revised; SCL-90-R; Derogatis, 1992), versión española realizada por González de Rivera et al. (1989) y sus propiedades psicométricas estudiadas por De Las Cuevas et al. (1991). Se trata de un cuestionario que interroga al individuo sobre la existencia e intensidad de 90 síntomas de malestar psicológico y psicosomático, valorándose la intensidad de cada síntoma en una escala tipo Likert, desde la ausencia total (0) hasta su máxima intensidad (4). Los ítems teóricamente se agrupan en 9 escalas, aunque los estudios factoriales no encuentran que los ítems se agrupen en ellas, representando más bien síntomas de malestar psicológico, y ello tanto en población clínica (De Las Cuevas et al., 1991) como en población clínica de adictos a sustancias (Pedrero Pérez y López-Durán, 2005). Cuenta con tres índices generales: Índice General Sintomático (IGS, intensidad del sufrimiento psíquico y psicosomático global), Total de Síntomas Positivos (TSP) e Índice de Malestar (PSDI, intensidad sintomática media). En el presente estudio, el SCL-90-R mostró una consistencia interna de $\alpha=0,97$, con todos los ítems menos uno (ítem 60) con una correlación ítem test corregida $0,30<$ rit $<0,71$.

Evaluación Cognitiva de Montreal (Montreal Cognitive Assessment, MoCA; Nasreddine et al, 2005), versión española propuesta por los propios autores y validada en población clínica de adictos a sustancias en España (Rojo-Mota, Pedrero-Pérez, Ruiz-Sánchez de León, Llanero-Luque y Puerta-García, 2013). Se trata de una prueba de cribado que valora 10 dominios cognitivos, a partir de formas básicas de tests neuropsicológicos ampliamente validados. La puntuación máxima es de 30 puntos, aunque se realiza una corrección con incremento de 2 puntos para sujetos con menos de 9 años de escolaridad y 1 si el tiempo de escolarización es entre 9 y 12 años (Chertkow, Nasreddine, Johns, Phillips y McHenry, 2011). Estudios transculturales estiman una puntuación de corte de 26, considerándose que quienes obtienen ésta o superiores puntuaciones presentan un rendi- miento normal, y puntuaciones menores sugieren sospecha de deterioro cognitivo leve o demencia temprana. El tiempo de administración es de 10 minutos aproximadamente. La consistencia interna de la prueba en el presente estudio fue de $\alpha=0,70$, con $0,30<$ rit $<0,46$ para los ítems.

Se utilizó el método de espectrofotometría ultravioleta visible para la determinación de metabolitos de opiáceos, cocaína, cannabis y benzodiacepinas en orina. En el caso de las benzodiacepinas, sólo se consideró positivo cuando no existía prescripción médica de alguna de ellas.

Para la obtención de los datos clínicos y sociodemográficos se consultó la historia clínica de cada sujeto. Se tuvo en cuenta el tiempo de permanencia en el programa actual, la edad, el sexo, el nivel de estudios y la dosis de metadona prescrita en el momento de la evaluación.

\section{Procedimiento}

Los evaluadores recibieron 3 sesiones de entrenamiento previas al inicio del periodo de evaluación (1 sesión presencial en el caso de Extremadura), además de asesoramiento permanente para la resolución de cuantas dudas surgieran posteriormente. Previamente a la administración del protocolo de evaluación se anunció mediante carteles en los despachos de dispensación sobre la intención de realizar el estudio y la voluntariedad en la participación. También se administraron folletos en los que se anunciaba el estudio. A partir del inicio del periodo de evaluación, se ofrecía a los pacientes la posibilidad de realizar el estudio en el momento en que acudían a recibir sus dosis (diarias o semanales). En caso de que no tuvieran disponibilidad de tiempo en ese momento, se les ofrecía una cita programada para realizarlo en los días siguientes. Un porcentaje bajo de los pacientes rechazó participar en el estudio $(n=70 ; 3,7 \%)$. En cuanto a los autoinformes, el evaluador leía las preguntas y el paciente señalaba las respuestas en unas fichas preparadas con las diferentes modalidades de respuesta. La prueba de rendimiento cognitivo se realizaba in situ, tras los autoinformes. Si el paciente solicitaba un descanso, se le ofrecía la posibilidad de realizarlo. Las evaluaciones duraban entre 30 y 45 minutos. Al finalizar, se solicitaba una muestra de orina para realizar análisis toxicológico y se advertía al paciente de que se recogería una segunda muestra una vez transcurrido un mes desde la primera, con independencia de las que se recogieran rutinariamente como parte de su tratamiento. Los protocolos cumplimentados se remitían por correo interno al investigador principal, que centralizaba los datos, configurando la base de datos. Se rechazaban los protocolos mal cumplimentados, con ausencia de datos o respuestas sin cumplimentar $(n=80)$. Para el estudio de las relaciones con otras variables se estimó la dosis percibida como variable lineal, y también se dividió a los participantes en grupos: dosis muy bajas (<30 mg/día), bajas (30-59 mg/día), medias (60$90 \mathrm{mg} /$ día $)$ y altas $(>90 \mathrm{mg} /$ día $)$. 
Todos los participantes recibieron información sobre el objetivo de la evaluación y firmaron un consentimiento informado, admitiendo la utilización anónima de los resultados. El estudio recibió la aprobación de los Comités de Ética de Cáceres y Badajoz.

\section{Análisis de datos}

Para las comparaciones entre categorías se utilizó la $\chi_{\mathrm{gl}}^{2}$. Para comparaciones entre variables continuas se utilizó la distribución $\mathrm{F}_{\mathrm{gl}}$ de Snedecor, mediante análisis de la varianza univariante o multivariante. La estimación de proporciones de varianza explicadas por covariables se estimó mediante la lambda de Wilks $(\lambda)$. Las correlaciones, lineales o parciales, se realizaron mediante la $r$ de Pearson. Se efectuaron análisis de regresión lineal por pasos sucesivos, reportando la proporción de varianza explicada $\left(\mathrm{R}^{2}\right)$ y el coeficiente $\beta$. Para el tamaño del efecto se utilizó el estimador eta al cuadrado $\left(\eta^{2}\right.$; Cohen, 1973) y para su interpretación se utilizaron las rules of thumb propuestas por el autor (Cohen, 1988): entre 0,01 y 0,06 efecto pequeño, entre 0,06 y 0,13 , efecto moderado y más de 0,13 efecto grande. Para las comparaciones categoriales se utilizó la V de Cramer como estimador del tamaño del efecto y para las correlaciones el coeficiente de mutua determinación $\left(\mathrm{r}^{2}\right)$. Para todos los análisis se utilizó el paquete estadístico SPSS 19 (el cálculo de $\eta^{2}$ se realizó manualmente). Se exploraron las relaciones estructurales entre las variables, mediante el método de máxima verosimilitud, y se compararon los diferentes modelos mediante índices de calidad de ajuste (ECVI, Hoelter), aplicando posteriormente índices de ajuste absolutos $\left(\chi^{2}\right.$, grados de libertad), relativos (CMIN/DF, RMSEA) e incrementales (NFI, CFI, RFI, IFI, TLI), siguiendo las recomendaciones de Hooper, Coughlan y Mullen (2008), a partir de la información proporcionada por el programa AMOS 18.

\section{Resultados}

\section{Descriptivos}

En la Tabla 1 se muestran los descriptivos de la muestra principal. Se observa una ratio de 4 varones por cada mujer en tratamiento, algo que es general en todos los países del entorno cultural y estable en el tiempo a lo largo de décadas. Analizado por sexos, los varones presentan una edad media significativamente mayor, aunque el tamaño del efecto de estas diferencias es insignificante $\left(\eta^{2}=0,01\right)$. Hay también diferencias significativas (aunque también con muy pobre tamaño del efecto $\mathrm{V}=0,03$ ) en el nivel formativo, siendo más frecuente que las mujeres se sitúen en los grupos extremos (menos de 9 años o más de 15), mientras que más de la mitad de los varones están comprendidos en el grupo de entre 9 y 12 años de escolarización. No aparecen diferencias significativas en cuanto a la dosis de metadona prescrita por sexos $\left(\mathrm{F}_{1}=0,02 ; \mathrm{p}=0,89\right)$. Tampoco aparece relación entre dosis prescrita y edad del paciente $(\mathrm{r}=0,06 ; \mathrm{p}=0,19)$, aunque sí con el tiempo de tratamiento $(r=0,10 ; p<0,05)$.

La muestra B se compuso de 57 varones y 8 mujeres, con una media de edad de 42,5 años (DT=7,1). El 36,9\% tenía menos de 9 años de escolarización, el 44,6\% entre 9 y 12 años, el 16,9\% entre 12 y 15 años y un 1,5\% más de 15 años (ninguna mujer entre las dos últimas categorías, el $75 \%$ menos de 9 años).

\section{Dosis}

Un $72,7 \%$ de la muestra recibía dosis inferiores a $60 \mathrm{mg} /$ día: el $37,3 \%$ menos de $30 \mathrm{mg} /$ día $(\mathrm{M}=15,1 ; \mathrm{DT}=7,2)$ y el $35,4 \%$ entre 30 y $60 \mathrm{mg} /$ día $(\mathrm{M}=41,6 ; \mathrm{DT}=8,2)$. Un $14,6 \%$ recibía entre 60 y $90 \mathrm{mg} /$ día $(\mathrm{M}=73,1 ; \mathrm{DT}=9,7)$ y sólo un 12,7\% recibía más de $90 \mathrm{mg}$ / día $(\mathrm{M}=126,1 ; \mathrm{D}=33,1)$.

La muestra B presentó características muy diferentes. Un $96,9 \%$ de la muestra recibía dosis inferiores a $60 \mathrm{mg}$ /día: el

Tabla 1. Descriptivos de la muestra.

\begin{tabular}{|c|c|c|c|c|c|}
\hline & Varones & Mujeres & Total & $F$ & $p$ \\
\hline $\mathrm{n}$ & 364 & 94 & 458 & & \\
\hline$\%$ & 79,5 & 20,5 & & & \\
\hline Edad media actual (d.t.) en años & $47,6(6,2)$ & $46,1(6,5)$ & $47,3(6,3)$ & 3,97 & $<0,05$ \\
\hline Años de escolarización & $\%$ & & & $\chi^{2}$ & $p$ \\
\hline$<9$ & 28,8 & 36,2 & & 9,1 & $<0,05$ \\
\hline $9-12$ & 51,1 & 34,0 & & & \\
\hline $12-15$ & 16,5 & 24,5 & & & \\
\hline \multirow[t]{2}{*}{$>15$} & 3,6 & 5,3 & & & \\
\hline & & & & $F$ & $p$ \\
\hline Dosis media (d.t.) en mg/día & $47,4(39,0)$ & $45,7(36,2)$ & $47,0(38,4)$ & 0,14 & 0,71 \\
\hline Rango & $5-220$ & $5-160$ & $5-220$ & & \\
\hline Tiempo medio de tto en meses (d.t.) & $93,3(120,3)$ & $89,5(67,1)$ & $92,5(111,4)$ & 0,09 & 0,77 \\
\hline Rango en meses & $3-2011$ & $3-281$ & $3-2011$ & & \\
\hline
\end{tabular}


$78,5 \%$ menos de $30 \mathrm{mg} /$ día $(\mathrm{M}=17,8 ; \mathrm{DT}=8,4)$ y el $18,5 \%$ entre 30 y $60 \mathrm{mg} /$ día $(\mathrm{M}=38,5 ; \mathrm{DT}=9,5)$. Un 3,1\% recibía entre 60 y $90 \mathrm{mg} /$ día $(\mathrm{M}=82,0 ; \mathrm{DT}=8,5)$ y nadie recibía más de $90 \mathrm{mg} /$ día.

\section{Relación dosis/calidad de vida autopercibida}

La dosis administrada correlacionó negativa y significativamente con la calidad de vida: dominio físico $(r=-0,24$; $\left.\mathrm{p}<0,001 ; \mathrm{r}^{2}=0,06\right)$, psicológico $\left(\mathrm{r}=-0,14 ; \mathrm{p}<0,01 ; \mathrm{r}^{2}=0,02\right)$, social $\left(\mathrm{r}=-0,10 ; \mathrm{p}<0,05 ; \mathrm{r}^{2}=0,01\right)$, ambiental $(\mathrm{r}=-0,19 ; \mathrm{p}<$ 0,$\left.001 ; r^{2}=0,04\right)$ y con la puntuación global $(r=-0,22 ; p<$ 0,$\left.001 ; r^{2}=0,05\right)$.

En la Tabla 2 se muestran los valores obtenidos en los diferentes dominios de la calidad de vida autopercibida según la dosis de metadona prescrita. Las puntuaciones mostraron diferencias significativas, tanto en la puntuación total de calidad de vida, como en cada uno de los dominios, siempre en dirección a una peor calidad de vida a medida que se consideraban dosis superiores. El tamaño del efecto de estas diferencias fue bajo, pero especialmente significativo en los dominios de salud física y ambiental, así como en la calidad de vida global. Las pruebas post hoc mostraron que las principales diferencias en salud física y ambiental se establecieron entre quienes tomaban dosis muy bajas y quienes recibían dosis medias o altas; en el dominio psicológico, entre los grupos extremos; y en relaciones sociales, entre los que tomaban dosis muy bajas y los que recibían dosis bajas.

Se estudió a continuación el posible efecto de otras variables sobre estas diferencias. Ni el sexo $\left(\lambda=0,99 ; \mathrm{F}_{4 ; 447}=0,74\right.$; $\mathrm{p}=0,56)$ ni el tiempo de tratamiento en programa $(=0,99$; $\left.\mathrm{F}_{4 ; 447}=0,64 ; \mathrm{p}=0,63\right)$ explicaron una parte significativa de la varianza de las diferencias observadas. Por el contrario, sí lo hacían tanto la edad $\left(\lambda=0,98 ; \mathrm{F}_{4 ; 447}=2,47 ; \mathrm{p}=0,04 ; \eta^{2}=\right.$
$0,022)$ como el nivel de estudios $\left(\lambda=0,96 ; \mathrm{F}_{4 ; 47}=5,13 ; \mathrm{p}<\right.$ 0,$\left.001 ; \eta^{2}=0,044\right)$. El efecto de la edad era significativo en el dominio ambiental $\left(\mathrm{F}_{1}=4,02 ; \mathrm{p}<0,05 ; \eta^{2}=0,009\right)$, en el social $\left(\mathrm{F}_{1}=7,86 ; \mathrm{p}<0,01 ; \eta^{2}=0,017\right)$ y en la puntuación global $\left(\mathrm{F}_{1}=4,17 ; \mathrm{p}<0,05 ; \eta^{2}=0,009\right)$, mientras que el nivel de estudios intervenía significativamente en los dominios psicológico $\left(\mathrm{F}_{1}=9,67 ; \mathrm{p}<0,01 ; \eta^{2}=0,021\right)$ y ambiental $\left(\mathrm{F}_{1}=15,04\right.$; $\left.\mathrm{p}<0,001 ; \eta^{2}=0,032\right)$, y también en la puntuación global $\left(\mathrm{F}_{1}=\right.$ 9,$\left.27 ; \mathrm{p}<0,01 ; \eta^{2}=0,020\right)$. Controlando el nivel de estudios, la edad correlacionó significativamente con las relaciones sociales $\left(\mathrm{r}=-0,13 ; \mathrm{p}<0,01 ; \mathrm{r}^{2}=0,02\right)$, con la calidad ambiental $\left(\mathrm{r}=-0,10 ; \mathrm{p}<0,05 ; \mathrm{r}^{2}=0,01\right)$ y con la puntuación global de calidad de vida $\left(\mathrm{r}=-0,10 ; \mathrm{p}<0,05 ; \mathrm{r}^{2}=0,01\right) ; \mathrm{y}$, controlando la edad, el nivel de estudios correlacionó significativamente con la salud psicológica $\left(\mathrm{r}=0,15 ; \mathrm{p}<0,01 ; \mathrm{r}^{2}=0,02\right)$, con la calidad ambiental $\left(\mathrm{r}=0,19 ; \mathrm{p}<0,001 ; \mathrm{r}^{2}=0,04\right)$ y con la puntuación global $\left(\mathrm{r}=0,15 ; \mathrm{p}<0,01 ; \mathrm{r}^{2}=0,02\right)$. En todos los casos, el tamaño del efecto fue bajo.

Cuando se estudiaron las diferencias según el grupo de dosis asignada y controlando las variables que previamente habían mostrado efecto de interacción (edad y nivel de estudios) aparecieron diferencias significativas en todos los dominios de salud (Tabla 2). Quienes recibían dosis más bajas (<30 mg/día) mostraban mayores niveles de salud, que iban descendiendo a medida que los grupos recibían dosis mayores. El tamaño del efecto de estas diferencias fue moderado en los casos de la salud física $\left(\eta^{2}=0,06\right)$, de la salud ambiental $\left(\eta^{2}=0,08\right)$ y de la puntuación global de calidad de vida $\left(\eta^{2}=0,08\right)$.

En la muestra B, la dosis correlacionó negativamente con la calidad de vida y todas sus dimensiones (salud física $\mathrm{r}=$ $-0,17$; psicológica $r=-0,23$; relaciones sociales $r=-0,07$; ambiental $r=-0,06$; puntuación global $r=-0,16$ ), aunque en ningún caso se alcanzó la significación estadística.

Tabla 2. Dominios de la calidad de vida (WHOQOL-BREF) y dosis de metadona prescrita.

\begin{tabular}{|c|c|c|c|c|c|c|c|}
\hline & \multicolumn{4}{|c|}{ Dosis } & & & \multirow[b]{2}{*}{$(*)$} \\
\hline & muy bajas & bajas & medias & altas & & & \\
\hline WHOQOL & \multicolumn{4}{|c|}{ M (DT) } & $\mathrm{F}_{\mathrm{df}=3}$ & $\eta^{2}$ & $\eta^{2}$ \\
\hline Salud física & $24,35(4,87)$ & $23,15(4,41)$ & $22,28(4,94)$ & $20,98(4,50)$ & 8,63 & 0,054 & 0,055 \\
\hline Total & \multicolumn{4}{|c|}{$23,20(4,79)$} & & & \\
\hline Salud psicológica & $18,96(4,40)$ & $18,62(4,16)$ & $18,52(4,14)$ & $17,05(4,35)$ & 2,94 & 0,019 & 0,042 \\
\hline Total & \multicolumn{4}{|c|}{$18,53(4,30)$} & & & \\
\hline Salud social & $9,19(2,55)$ & $8,41(2,61)$ & $8,64(2,37)$ & $8,21(2,59)$ & 3,52 & 0,023 & 0,041 \\
\hline Total & \multicolumn{4}{|c|}{$8,71(2,57)$} & & & \\
\hline Salud ambiental & $26,52(4,73)$ & $25,35(4,76)$ & $24,54(5,25)$ & $23,62(5,50)$ & 6,17 & 0,039 & 0,077 \\
\hline Total & \multicolumn{4}{|c|}{$25,45(5,00)$} & & & \\
\hline Calidad de vida & $79,01(12,98)$ & $75,53(12,55)$ & $73,99(13,37)$ & $69,86(13,39)$ & 8,05 & 0,051 & 0,075 \\
\hline Total & \multicolumn{4}{|c|}{$75,89(13,24)$} & & & \\
\hline
\end{tabular}

Nota. ${ }^{\star}$ Controlando la edad y el nivel de estudios $\left(\mathrm{F}_{\mathrm{df}=5}\right)$. 


\section{Relación dosis/satisfacción con el tratamiento}

El 96,5\% de la muestra se declaró satisfecho $(50,2 \%)$ o muy satisfecho $(46,3 \%)$ con el tratamiento recibido y sólo un $3,5 \%$ se declaró moderadamente insatisfecho. No aparecieron diferencias significativas entre los diferentes grupos según la dosis de metadona prescrita $\left(\mathrm{F}_{3}=1,94 ; \mathrm{p}=0,12\right)$. Cuando se controló el efecto de las covariables se encontró significación en relación al sexo $\left(\mathrm{F}_{7}=5,43 ; \mathrm{p}<0,05 ; \eta^{2}=\right.$ $0,012)$ y a la edad $\left(F_{7}=10,86 ; p<0,01 ; 2=0,024\right)$, pero no en cuanto al nivel de estudios ni el tiempo de tratamiento. Las mujeres se mostraron significativamente $\left(\mathrm{F}_{1}=6,75 ; \mathrm{p}<0,05\right.$; $\left.\eta^{2}=0,015\right)$ más satisfechas $(\mathrm{M}=78,6 ; \mathrm{DT}=13,0)$ que los varones $(\mathrm{M}=74,9 ; \mathrm{DT}=12,4)$. La edad mostró correlación negativa con la satisfacción $\left(r=-0,17 ; \mathrm{p}<0,001 ; \mathrm{r}^{2}=0,03\right)$, incluso cuando se controló el sexo $\left(r=-0,16 ; p<0,01 ; r^{2}=0,03\right)$. Cuando ambas variables se controlaron, las diferencias entre los grupos por dosis alcanzaron significación, puntuando más alto en satisfacción con el tratamiento cuanto menor era la dosis de metadona administrada (Tabla 3).

En la muestra B, los niveles de satisfacción fueron similares: el 96,9\% se mostraron satisfechos o muy satisfechos con el tratamiento. La puntuación de satisfacción correlacionó negativamente con la dosis $(\mathrm{r}=-0,14)$, aunque no se alcanzó la significación estadística $(\mathrm{p}=0,27)$.

\section{Relación dosis/malestar psicológico}

En la Tabla 4 se observa que todos los índices del SCL90-R muestran un incremento paralelo a las dosis de metadona prescritas. Las pruebas post hoc mostraron que sólo el grupo de mayor dosis mostraba diferencias significativas con los demás, con más síntomas positivos y un mayor Índice General Sintomático. Cuando se estudió el posible efecto de otras variables sobre estas diferencias se observó que sólo el sexo mostraba efecto de interacción significativo $(\lambda=0,97$; $\left.\mathrm{F}_{3 ; 448}=2,47 ; \mathrm{p}<0,01 ; \eta^{2}=0,035\right)$, pero no la edad $(\lambda=0,99$; $\left.\mathrm{F}_{3 ; 448}=2,21 ; \mathrm{p}=0,09\right)$, el nivel de estudios $\left(\lambda=0,99 ; \mathrm{F}_{3 ; 448}=\right.$ $2,12 ; \mathrm{p}=0,10)$, ni el tiempo de tratamiento $\left(\lambda=0,99 ; \mathrm{F}_{3 ; 448}=\right.$ $1,23 ; \mathrm{p}=0,30)$. Las mujeres puntuaban significativamente más que los varones en los 3 índices: Índice General Sintomático $\left(\mathrm{F}_{\mathrm{gl}=1}=15,2 ; \mathrm{p}<0,001 ; \eta^{2}=0,032\right)$, Total de Síntomas Positivos $\left(\mathrm{F}_{\mathrm{gl}=1}=8,3 ; \mathrm{p}<0,01 ; \eta^{2}=0,018\right)$ e Intensidad Somática Media $\left(\mathrm{F}_{\mathrm{gl}=1}=14,5 ; \mathrm{p}<0,001 ; \eta^{2}=0,031\right)$. En la Tabla 4 se observa que la relación entre dosis y malestar es lineal en los varones, pero son las mujeres que toman dosis medias (60-90 mg/día) las que presentan mayores indicadores de malestar.

Se efectuó un análisis de regresión de las puntuaciones de las escalas del SCL-90-R sobre la dosis de metadona recibida para conocer qué grupos de síntomas se asociaban a mayores dosis. En varones, fue la escala de Somatización la

Tabla 3. Puntuaciones de satisfacción con el tratamiento según la dosis de metadona prescrita, controlando el sexo y la edad.

\begin{tabular}{|c|c|c|c|c|c|c|c|}
\hline & \multicolumn{4}{|c|}{ Dosis } & & & \\
\hline & muy bajas & bajas & medias & altas & & & \\
\hline \multirow[b]{2}{*}{ Satisfacción } & \multicolumn{4}{|c|}{ M (DT) } & $\mathrm{F}_{\mathrm{gl}=5}$ & Sig. & $\eta_{\mathrm{p}}^{2}$ \\
\hline & $77,14(12,89)$ & $75,64(11,65)$ & $74,14(13,25)$ & $73,08(13,05)$ & 4,8 & $p<0,001$ & 0,051 \\
\hline
\end{tabular}

Tabla 4. Índices de malestar del SCL-9o-R.

\begin{tabular}{|c|c|c|c|c|c|c|c|}
\hline \multirow[b]{4}{*}{ Índice General Sintomático } & \multicolumn{4}{|c|}{ Dosis } & \multirow[b]{3}{*}{$\mathrm{F}_{\mathrm{gl}=5}$} & \multirow[b]{3}{*}{ Sig. } & \multirow[b]{3}{*}{$\eta_{p}^{2}$} \\
\hline & muy bajas & bajas & medias & altas & & & \\
\hline & \multicolumn{4}{|c|}{$M(D T)$} & & & \\
\hline & $0,75(0,55)$ & $0,785(0,59)$ & $0,91(0,59)$ & $1,10(0,65)$ & 5,93 & $p<0,01$ & 0,038 \\
\hline Total Síntomas Positivos & $35,74(18,1)$ & $37,60(20,3)$ & $42,93(19,5)$ & $47,66(19,5)$ & 6,83 & $p<0,001$ & 0,043 \\
\hline \multirow[t]{2}{*}{ Índice General Sintomático } & $1,70(0,59)$ & $1,72(0,53)$ & $1,79(0,54)$ & $1,93(0,57)$ & 2,62 & $p=0,51$ & 0,017 \\
\hline & \multicolumn{4}{|c|}{ Varones } & $\mathrm{F}_{\mathrm{gl}=3}$ & Sig. & $\eta_{p}^{2}$ \\
\hline Índice General Sintomático & $0,71(0,51)$ & $0,73(0,56)$ & $0,79(0,51)$ & $1,10(0,68)$ & 6,13 & $p<0,001$ & 0,049 \\
\hline Total Síntomas Positivos & $34,7(17,3)$ & $36,4(20,1)$ & $39,9(18,7)$ & $47,3(19,2)$ & 5,58 & $p<0,01$ & 0,044 \\
\hline \multirow[t]{2}{*}{ Índice General Sintomático } & $1,68(0,56)$ & $1,64(0,51)$ & $1,68(0,47)$ & $1,93(0,60)$ & 3,28 & $p<0,05$ & 0,027 \\
\hline & \multicolumn{4}{|c|}{ Mujeres } & $\mathrm{F}_{\mathrm{gl}=3}$ & Sig. & $\eta_{p}^{2}$ \\
\hline Índice General Sintomático & $0,90(0,65)$ & $1,03(0,67)$ & $1,35(0,69)$ & $1,10(0,57)$ & 1,65 & $p=0,18$ & 0,052 \\
\hline Total Síntomas Positivos & $39,8(20,6)$ & $42,6(20,9)$ & $54,4(18,5)$ & $48,8(16,2)$ & 2,11 & $p=0,10$ & 0,066 \\
\hline Índice General Sintomático & $1,80(0,67)$ & $2,02(0,53)$ & $2,17(0,62)$ & $1,93(0,63)$ & 1,48 & $p=0,23$ & 0,047 \\
\hline
\end{tabular}


única que presentó capacidad predictiva positiva $\left(\mathrm{R}^{2}=0,06\right.$; $\beta=14,6)$, mientras que en mujeres fue la escala de Ansiedad Fóbica $\left(R^{2}=0,05 ; \beta=13,6\right)$. Cuando se efectuó el mismo procedimiento a partir de los ítems del SCL-90-R (Tabla 5), se encontraron modelos que explicaban una parte significativa de la varianza de las dosis (12\% en varones, $17 \%$ en mujeres), pero ninguno de los modelos resultaba adecuado al generar un excesivo número de residuos (Durbin-Watson < 1 , en ambos casos).

En la muestra B, la dosis correlacionó negativamente con todas las escalas e índices del SCL-90-R, aunque en ningún caso se alcanzó la significación estadística.

\section{Relación dosis/rendimiento cognitivo}

Sólo el $40 \%$ de la muestra presenta un rendimiento cognitivo normal (puntuación en el MoCA $\geq 26$ ), en tanto que un $41,5 \%$ presenta un deterioro cognitivo leve (entre 21 y $25)$ y un $18,5 \%$ niveles más graves de afectación $(\leq 20)$. Tomando la puntuación del MoCA como variable continua, no aparecen diferencias en el rendimiento cognitivo según el grupo de dosis recibidas $\left(\mathrm{F}_{\mathrm{gl}=3}=1,96 ; \mathrm{p}=0,12\right)$, ni efecto de las variables sexo $\left(\mathrm{F}_{\mathrm{gl}=1}=0,00 ; \mathrm{p}=0,99\right)$, edad $\left(\mathrm{F}_{\mathrm{gl}=1}=0,08 ; \mathrm{p}=\right.$ $0,77)$ o tiempo de tratamiento $\left(\mathrm{F}_{\mathrm{gl}=1}=0,57 ; \mathrm{p}=0,45\right)$. El nivel de escolarización no muestra efecto de interacción cuando se toman las puntuaciones corregidas $\left(\mathrm{F}_{\mathrm{gl}=1}=2,54 ; \mathrm{p}=0,11\right)$, pero sí cuando se utilizan las puntuaciones en bruto, sin corrección $\left(\mathrm{F}_{\mathrm{gl}=1}=24,99 ; \mathrm{p}<0,001 ; \eta^{2}=0,055\right)$. Sin embargo, cuando la dosis se toma como variable continua, aparece una relación significativa y negativa entre dosis y puntuación obtenida en el MoCA $\left(r=-0,22 ; p<0,001 ; r^{2}=0,05\right)$, que se mantiene en los mismos niveles cuando se controla el resto de variables.

Cuando se clasifica a los sujetos según el rendimiento en el MoCA (normal, deterioro leve y grave), aparecen diferencias significativas (Tabla 6). Sólo un 25,9\% de quienes toman más de $90 \mathrm{mg}$ /día de metadona y un 25,4\% de quienes reciben $60-90 \mathrm{mg} /$ día presentan un rendimiento cognitivo normal, mientras que este porcentaje es del 50,3\% para quienes toman dosis muy bajas y del $40,1 \%$ para quienes reciben dosis entre $30-60 \mathrm{mg} /$ día.

En la muestra B, el 32,3\% de los sujetos mostró un rendimiento cognitivo normal, el $50,8 \%$ un deterioro leve y el $16,9 \%$ un deterioro grave. No se encontró correlación significativa entre la puntuación en el MoCA y la dosis de metadona administrada, y tampoco se obtuvo significación estadística entre estas variables, ni siquiera cuando se controlaron el resto de variables.

\section{Relación dosis/consumo de sustancias no prescritas}

En el momento de la evaluación, un 14,2\% de los sujetos presentaban un resultado positivo para opiáceos (diferentes de la metadona), un $24,5 \%$ para cocaína, un $34,9 \%$ para cannabis y un $9,0 \%$ para benzodiacepinas no prescritas. Transcurrido al menos un mes desde el momento de la evaluación, los resultados positivos fueron un $13,8 \%$ para opiáceos, un $23,1 \%$ para cocaína, un $33,2 \%$ para cannabis y un

Tabla 5. Ítems del SCL-9o-R con capacidad predictiva sobre la dosis de metadona.

\begin{tabular}{llcc}
\hline Ítem & Varones & $\mathrm{R}^{2} \times 100$ & \multicolumn{1}{c}{$\beta$} \\
\hline 58 & Sensaciones de pesadez en sus brazos o piernas & 4,67 & 5,96 \\
75 & Sentirse nervioso cuando le dejan solo & 2,99 & 8,54 \\
12 & Dolores en el pecho o en el corazón & 1,32 & 5,35 \\
24 & Explosiones temperamentales que no puede controlar & 1,65 & $-6,68$ \\
61 & Sentirse incómodo cuando la gente le mira o habla de usted & 1,52 & 5,07 \\
& Mujeres & & 10,54 \\
82 & Sentir miedo de desmayarse en público & 3,21 & 13,12 \\
88 & No sentirse nunca cercano o compenetrado con otra persona & 2,86 & $-9,75$ \\
33 & Sentirse temeroso & 6,58 \\
\hline
\end{tabular}

Tabla 6. Porcentaje de sujetos según el tipo de rendimiento en el MoCA y según dosis de metadona.

\begin{tabular}{|c|c|c|c|c|c|c|}
\hline \multirow[b]{3}{*}{ MoCA } & \multicolumn{4}{|c|}{ Dosis } & \multirow[b]{3}{*}{$\chi_{6}^{2}$} & \multirow[b]{3}{*}{ Sign. } \\
\hline & muy bajas & bajas & medias & altas & & \\
\hline & \multicolumn{4}{|c|}{ Porcentaje de sujetos } & & \\
\hline Deterioro grave & $14,6 \%$ & $19,1 \%$ & $16,4 \%$ & $31,0 \%$ & 23,8 & $p<0,01$ \\
\hline Deterioro leve & $35,1 \%$ & $40,7 \%$ & $58,2 \%$ & $43,1 \%$ & & \\
\hline Rendimiento normal & $50,3 \%$ & $40,1 \%$ & $25,4 \%$ & $25,9 \%$ & & \\
\hline
\end{tabular}


9,6\% para benzodiacepinas. Considerando las dos tomas, un $81,9 \%$ de los sujetos mostraron abstinencia a la heroína (8,3\% positivo en uno de los análisis y $9,8 \%$ positivo en ambos), un $71,2 \%$ mostraron abstinencia a la cocaína (10,0 positivo en un análisis, 18,8 positivo en ambos), un 60,0\% mostraron abstinencia completa a cannabis $(11,8 \%$ positivo en un análisis, $28,2 \%$ positivo en ambos) y un $88,2 \%$ mostraron abstinencia a benzodiacepinas no prescritas $(5,0 \%$ positivo en un análisis, $6,8 \%$ positivo en ambos). Un 41,3\% resultaron negativos para todas las sustancias en ambos controles.

Cuando se estudiaron los resultados según la dosis recibida, no aparecieron diferencias significativas (Tabla 7). Tampoco resultó significativa la diferencia en los positivos a opiáceos cuando sólo consideramos los grupos extremos, de dosis muy bajas y dosis altas en la primera toma $\left(\chi_{1}^{2}=\right.$ $3,56 ; \mathrm{p}=0,06)$, pero sí en la segunda $\left(\chi_{1}^{2}=5,96 ; \mathrm{p}<0,05 ; \mathrm{V}=\right.$ $0,02)$, siendo significativamente mayor entre quienes toman menos de $30 \mathrm{mg}$ /día que en quienes reciben más de $90 \mathrm{mg}$ / día de metadona. El número de sujetos que dio resultado negativo a opiáceos en ambas pruebas fue también superior en el grupo de altas dosis que en el de quienes tomaban menos de $30 \mathrm{mg} /$ día $\left(\chi_{1}^{2}=6,00 ; \mathrm{p}<0,05 ; \mathrm{V}=0,02\right)$, en el de quienes tomaban entre $30-60 \mathrm{mg} /$ día $\left(\chi_{1}^{2}=6,00 ; \mathrm{p}<0,05\right.$; $\mathrm{V}=0,02)$ y entre quienes tomaban entre $69-90 \mathrm{mg} /$ día $\left(\chi_{1}^{2}=\right.$ $4,14 ; \mathrm{p}<0,05 ; \mathrm{V}=0,02)$. No hubo ninguna diferencia significativa en el caso del resto de drogas buscadas en orina. Tampoco hubo diferencias significativas en relación al sexo, ni al tiempo de escolarización, ni al tiempo de tratamiento en ninguna sustancia rastreada.

En la muestra B, un $27,7 \%$ tenía restos de opiáceos diferentes de la metadona en el momento de la evaluación (26,2\% en la segunda prueba), un $27,7 \%$ tenía restos de cocaína $(23,1 \%$ en la segunda prueba), un $50,8 \%$ de cannabis (mismo valor en la segunda) y un $15,4 \%$ de benzodiacepinas $(13,8 \%$ en la segunda). Un $36,9 \%$ de la muestra obtuvo resultados negativos para todas las drogas en ambas pruebas. En ningún caso se encontró relación con la dosis de metadona recibida.

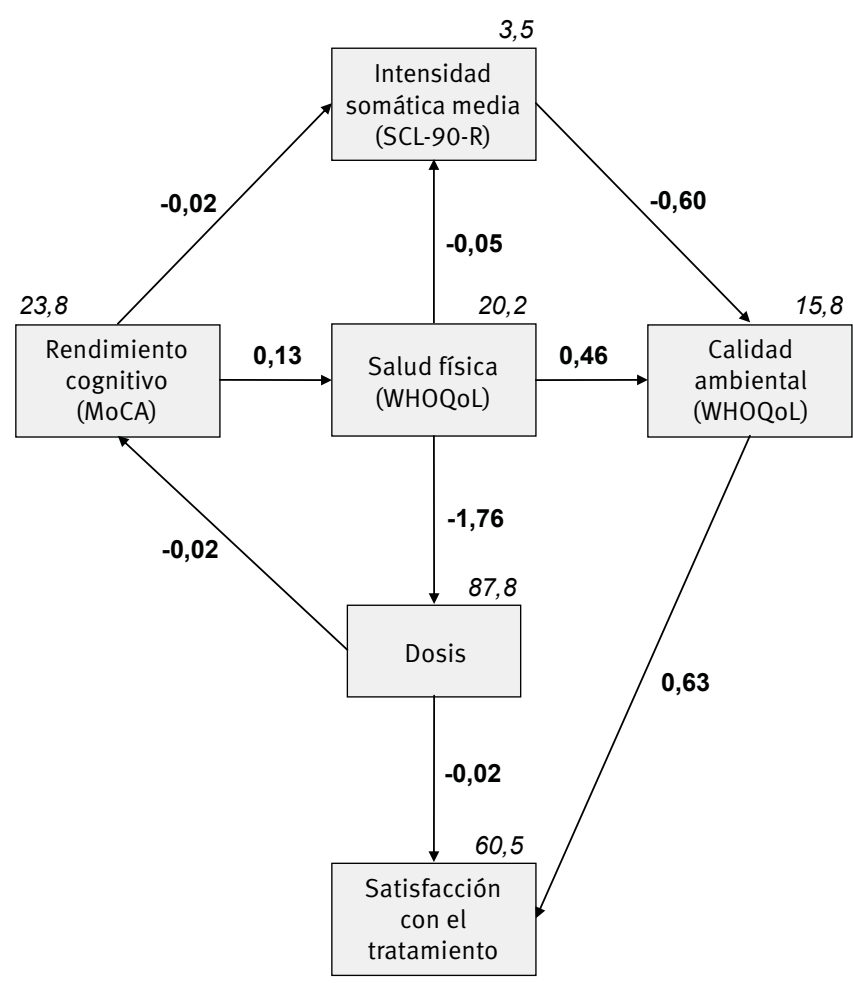

Figura 1. Modelo estructural de relación entre variables.

\section{Modelo estructural de las relaciones entre variables}

Finalmente, y a partir de las relaciones encontradas entre las diferentes variables, se hipotetizaron diversos modelos de relación estructural entre ellas. El modelo que alcanzó mejores indicadores de ajuste $(\mathrm{ECVI}=0,11$; Hoelter $=914$; $\mathrm{p}=0,05$,) fue el que se muestra en la Figura 1. Todos los indicadores mostraron un buen ajuste a los datos. $\left(\chi^{2}=6,3\right.$; g.l. $=6 ; \mathrm{p}=0,39 ; \mathrm{CMIN} / \mathrm{DF}=1,05 ; \mathrm{RMSEA}=0,01 ; \mathrm{NFI}=0,98$; $\mathrm{CFI}=0,99 ; \mathrm{RFI}=0,96 ; \mathrm{IFI}=0,99 ; \mathrm{TLI}=0,99$ ).

Tabla 7. Porcentaje de controles toxicológicos positivos para cada droga según dosis de metadona prescrita.

\begin{tabular}{|c|c|c|c|c|c|c|}
\hline \multirow[b]{3}{*}{ 1ํㅡoma } & \multicolumn{4}{|c|}{ Dosis } & \multirow[b]{3}{*}{$\chi_{3}^{2}$} & \multirow[b]{3}{*}{ Sig. } \\
\hline & muy bajas & bajas & medias & altas & & \\
\hline & \multicolumn{4}{|c|}{$\%$ positivos } & & \\
\hline Opiáceos & 17,00 & 13,60 & 14,90 & 6,90 & 3,70 & $p=0,30$ \\
\hline Cocaína & 21,60 & 26,50 & 26,90 & 24,10 & 1,33 & $p=0,72$ \\
\hline Cannabis & 32,20 & 39,50 & 31,30 & 34,50 & 2,45 & $p=0,48$ \\
\hline Benzodiac. & 7,60 & 9,30 & 11,90 & 8,60 & 1,14 & $p=0,77$ \\
\hline \multicolumn{7}{|l|}{$2^{\mathrm{a}}$ Toma } \\
\hline Opiáceos & 15,80 & 16,00 & 11,90 & 3,40 & 6,70 & $p=0,08$ \\
\hline Cocaína & 21,60 & 24,10 & 22,40 & 25,90 & 0,56 & $p=0,91$ \\
\hline Cannabis & 31,60 & 36,40 & 34,30 & 27,60 & 1,82 & $p=0,61$ \\
\hline Benzodiac. & 6,40 & 11,10 & 11,90 & 12,10 & 3,23 & $p=0,36$ \\
\hline
\end{tabular}




\section{Discusión}

El tratamiento sustitutivo con metadona es la estrategia terapéutica de elección en casi todos los casos en que un paciente demanda ayuda profesional por dependencia de heroína, pero existen variables individuales, farmacológicas, sociales y culturales que pueden influir en el modo en que se proporciona este tratamiento. El presente trabajo ha tenido por objeto explorar la relación entre las dosis de metadona administradas y diversas variables relacionadas. El objetivo final es encontrar evidencia científica que oriente a los médicos prescriptores en la tarea de proporcionar las dosis más adecuadas.

El presente trabajo encuentra una relación lineal entre mayor dosis de metadona prescrita y una menor calidad de vida autopercibida, lo que afecta a todas las dimensiones de la evaluación subjetiva. En especial, quienes toman dosis mayores de $60 \mathrm{mg} /$ día estiman niveles de calidad de vida significativamente más bajos. El tamaño del efecto de estas diferencias fue especialmente significativo en los dominios de salud física y ambiental, así como en la calidad de vida global. Las pruebas post hoc mostraron que las principales diferencias en salud física y ambiental se establecieron entre quienes tomaban dosis muy bajas y quienes recibían dosis medias o altas; en el dominio psicológico, entre los grupos extremos. Cuando se controlaron el resto de variables, el tamaño del efecto de las diferencias fue moderadamente alto en lo que se refiere a la evaluación que los sujetos hacen de sus condiciones ambientales, y también en la calidad de vida considerada globalmente.

La satisfacción de los pacientes con el tratamiento que se les proporciona es otra forma de evaluación de la adecuación de los programas a sus problemas. Los porcentajes encontrados en el presente estudio apuntan a un acuerdo casi total entre las expectativas y necesidades de los pacientes y la atención que se les proporciona desde los servicios especializados participantes. Los resultados superan los obtenidos para el conjunto de la población española en tratamiento con metadona (Pérez de los Cobos et al., 2004). Sin embargo, también aparece una relación negativa entre dosis administrada y grado de satisfacción. Estos datos parecen contravenir la creencia, ampliamente extendida, de que los pacientes requieren dosis más altas de las necesarias, para sentir los efectos psicoactivos de la metadona. Más adelante se integrarán estos resultados con los obtenidos en otras variables.

Se encuentra también relación lineal entre dosis de metadona y malestar psicológico, especialmente en el caso de las personas que reciben dosis altas (>90 mg/día). Los síntomas más frecuentemente asociados a las dosis son de carácter somático e inespecífico, o bien de carácter ansioso en las mujeres. No parece existir un cuadro sintomatológico asimilable a categorías diagnósticas específicas, sino a un malestar inespecífico, como ya se ha observado en estudios precedentes (De Las Cuevas et al., 1991; Pedrero Pérez y
López-Durán, 2005). Cabe planterase si este malestar puede atribuirse a efectos secundarios de la metadona, o bien al hecho de que personas con mayores niveles de malestar soliciten incrementos de dosis de metadona en un intento por mitigarlos. Si se tratara de la segunda opción, los datos del presente estudio apuntan a la ineficacia del método, por lo que es más probable que sean los efectos secundarios de la metadona los que se incrementan a medida que se incrementa la dosis. No obstante, el tamaño del efecto encontrado es bajo en casi todos los casos, lo que hace poco relevante la relación entre dosis y malestar psicológico.

Sólo un $40 \%$ de pacientes mostró un rendimiento cognitivo adecuado, según las puntuaciones de corte propuestas para el MoCA. Esta cifra es menor que la obtenida en estudios con el mismo instrumento, en programas de altas dosis, que encuentran un 62\% (Copersino et al., 2012). Sin embargo, esta cifra es superior a la encontrada en el mismo ámbito asistencial cuando se evaluó a sujetos que iniciaban tratamiento por adicción a diversas drogas, que fue del 29,1\% (Rojo-Mota et al., 2013). Lo que estos datos sugieren es que el tratamiento con metadona mejora el rendimiento que pudiera esperarse en la línea base, cuando intervinieran factores como el estrés asociado a la conducta de consumo, pero el tratamiento no consigue aproximar a un buen número de pacientes a los valores poblacionales, ni siquiera al de quienes mantienen abstinencia a todos los opiáceos, incluida la metadona, tras un periodo de adicción (Darke et al., 2012). Y, adicionalmente, el deterioro cognitivo leve asociado al tratamiento con metadona mantiene una relación lineal con la dosis, como así indican los resultados del presente estudio: en torno a un $40-50 \%$ de quienes reciben dosis menores de $60 \mathrm{mg}$ /día presentan un rendimiento normal, el doble de quienes toman más de $60 \mathrm{mg}$. No se dispone de estudios que permitan comparar estos datos, principalmente por el hecho de que la mayor parte de los programas ha adoptado las políticas de dosis altas, desatendiendo la relación entre dosis y deterioro cognitivo, en favor de otros indicadores de resultados.

Como en todos los estudios publicados, existe un alto porcentaje de sujetos que persiste en el consumo de drogas durante el tratamiento con metadona. En el presente estudio, un $60 \%$ presentó algún resultado positivo en orina para cocaína, cannabis o heroína en el momento de la evaluación. La comparación con otros estudios es difícil: los métodos utilizados son dispares, siendo frecuente el autoinforme de consumo. Algunos estudios informan de cifras superiores al $70 \%$ de sujetos que han consumido alguna droga no prescrita el mes anterior, con un $67 \%$ habiendo usado heroína en la semana previa (Curran et al., 1999). El presente estudio no puede valorar la dimensión temporal del consumo, al contar sólo con una aproximación en el momento de las evaluaciones. En todo caso, las cifras previamente encontradas suelen ser muy superiores al 60\% de consumos no prescritos, (Darke et al., 2012; Dobler-Mikola et al., 2005). 
El 18,1\% de los sujetos presentó metabolitos de opiáceos diferentes de la metadona en ambas tomas. Podemos, pues, considerar que el $80 \%$ de la muestra no presenta un consumo continuado de heroína o se encuentra abstinente a esta droga. Este porcentaje es sensiblemente inferior al encontrado en otros estudios, aunque los diferentes métodos no permiten establecer un paralelismo completo (Keen, Oliver, Rowse y Mathers, 2003; Musshoff, Trafkowski, Lichtermann y Madea, 2010). Lo que sí se observa es que quienes reciben las mayores dosis presentan significativamente menos consumo de heroína que quienes recibían menos de $90 \mathrm{mg} /$ día. En cuanto a la cocaína, no aparecieron diferencias en función de las dosis. Estos datos difieren de lo encontrado en otros estudios, que encuentran menor consumo de heroína y mayor de cocaína en quienes reciben dosis altas, y al contrario, en quienes reciben dosis bajas (Baumeister et al., 2014).

$\mathrm{Si}$ todos los indicadores previos resultan más favorables cuanto menor es la dosis prescrita, el consumo de heroína apunta en la dirección contraria. La relación negativa entre dosis y riesgo de muerte por sobredosis es un hallazgo repetido en los estudios previos (Liao et al., 2013; Liu et al., 2013; Van Ameijden et al., 1999), pero esta relación sólo se confirma en el caso en que exista consumo inyectado de heroína añadido a la administración terapéutica de metadona. En este caso, los efectos sobre receptores opioides de ambas sustancias resultan sumatorios, algo que no sucede cuando el consumo es inhalado o intranasal.

Los resultados invitan a realizar algunas reflexiones. En primer lugar, nada parece apoyar la prescripción de dosis altas, salvo en el caso de que persista el consumo inyectado de heroína. En los últimos años hemos asistido a una insistencia de ciertas instituciones y grupos de investigación para proporcionar dosis en torno a los $100 \mathrm{mg} /$ día, con independencia de otras variables (individuales, ambientales, terapéuticas, etc.) diferentes de la mera dependencia de opiáceos. Para llegar a esta conclusión se han realizado multitud de estudios que prueban que ciertos indicadores mejoran con dosis altas: el incremento de la retención en programas, la reducción de la actividad delictiva o la disminución del consumo de otras drogas (Lingford-Hughes, Welch y Nutt, 2004). Sin embargo, muchos menos estudios han explorado variables relativas al paciente: su calidad de vida, su satisfacción con el tratamiento, su grado de reincorporación laboral o la remisión o recuperación de la conducta adictiva. Cuando se han efectuado revisiones sobre trabajos del máximo nivel, no se han encontrado suficientes estudios que exploren la perspectiva del paciente (Amato et al., 2005; Fernández Miranda, 2001). Se trata de un caso excepcional en el ámbito sanitario: prescindir de la perspectiva y la valoración del paciente es algo inaceptable en cualquier otro problema de salud. La razón de este desprecio hay que buscarla en la predominancia de un modelo de enfermedad cerebral de la adicción que convierte al adicto en incapaz de tomar decisiones adecuadas o verter juicios valorables, con un cerebro secuestrado por la droga (Leshner, 1997). Algunas voces se han levantado, denunciando que los servicios médicos han adoptado, en ocasiones, un rol de control social sobre estos pacientes, tenidos por incapaces de regular su comportamiento y las políticas sanitarias de dosis altas han favorecido la cronificación del trastorno y los tratamientos, convirtiendo al paciente en un mero recipiente del tratamiento (Harris y McElrath, 2012), agravando la estigmatización y provocando que muchas personas en tratamiento se vean obligadas a vivir en excesivo estado de sedación, impotencia, malestar físico y emocional, e incapaces de participar activamente en la vida cotidiana de su comunidad (Bourgois, 2000; Notley, Blyth, Maskrey, Pinto y Holland, 2015).

En los últimos años, el modelo de enfermedad cerebral está siendo fuertemente contestado, evidenciando que ninguno de los objetivos que perseguía han llegado a cumplirse, incrementando la estigmatización social y obligando a la inmensa mayoría de los adictos a recibir tratamientos que sólo tendrían sentido para los pocos casos de mayor gravedad (Hall, Carter y Forlini, 2015; Hammer et al., 2013). El bloqueo químico de los receptores deposita el control sobre el médico y deja de lado intervenciones que persigan la toma de control del propio sujeto sobre su conducta. Cuando la dosis no produce aletargamiento, la persistencia en el consumo o el éxito del tratamiento dependen de variables psicológicas (Senbanjo, Wolff, Marshall y Strang, 2009; Zeldman et $a l ., 2004)$, la satisfacción del paciente con el tratamiento recibido es el mejor predictor de resultados (Kelly et al., 2011), los resultados dependen más de la provisión de servicios psicosociales como complementos del tratamiento farmacológico (Mino et al., 1998), determinadas intervenciones psicoterapéuticas reducen las dosis necesarias (Preston, Umbricht y Epstein, 2000), las dosis bajas se muestran tan útiles como las altas cuando se combinan con tratamientos psicosociales (Langendam, Van Brussel, Coutinho y Van Ameijden, 2001) y los pacientes son capaces de autogestionar sus dosis por encima de las imposiciones de los programas (Harris y Rhodes, 2013). Este enfoque se corresponde con un estilo de provisión de cuidados orientado a la recuperación, y no al control médico/social (White y Mojer-Torres, 2010).

El estudio presentado tiene limitaciones, algo común a la mayor parte de los trabajos similares consultados. Es imposible atender a todas las variables implicadas en un tratamiento en medio natural. Por ejemplo, muchos pacientes, pero no todos, reciben fármacos psicoactivos como complemento para reducir problemas psicopatológicos. Los efectos de estos fármacos pueden tener relación con la calidad de vida, la satisfacción y el rendimiento cognitivo, en uno u otro sentido. No se ha controlado el consumo crónico de alcohol (aunque sí la dependencia), que puede interferir de manera importante en el rendimiento cognitivo (Chen et al., 2011). Lo mismo sucede con las benzodiazepinas, que sólo han podido ser controladas cuando no han sido prescritas, pero pueden tomarse en dosis mayores a las prescritas. En general, el con- 
sumo de sustancias diferentes de la metadona se ha circunscrito al momento de la evaluación y a un punto más allá del mes posterior, pero esto no nos informa de la intensidad, cronicidad y variedad de patrones de consumo, si bien se han excluido aquellos pacientes con dependencia contrastada de alguna de ellas. Todos los participantes disponen de intervenciones diferentes a la médica: psicológicas, ocupacionales, integración social y laboral, cuidados de enfermería; pero no todos hacen el mismo uso de ellas, ni presentan la misma adherencia al tratamiento, no pudiéndose cuantificar el impacto de cada una de ellas o su papel en los resultados obtenidos.

En conclusión, nuestros datos apoyan el uso de dosis ajustadas a las necesidades individuales de cada paciente, mediante la negociación médico-paciente y la valoración dinámica de cada caso. Con este enfoque no se consigue la abstinencia completa, pero tampoco se obtiene en los programas de altas dosis, como se ha visto en la revisión bibliográfica, a pesar de que sea una de sus justificaciones principales y lo pretenda a partir de un bloqueo completo de los receptores opioides. No obtante, el consumo de sustancias no prescritas es menor que en otros estudios, aunque persiste en una mayoría de los pacientes. Los niveles de calidad de vida autopercibida son aceptables y, cuando menos, comparables a los obtenidos en programas de diferente orientación, si bien el hecho de permanecer en un tratamiento es una barrera para alcanzar los niveles normales en su medio habitual. La satisfacción de los usuarios es difícilmente mejorable, lo que indica una plena aceptación del modelo individualizado orientado a la recuperación. La percepción de salud física y su relación con la salud ambiental son las claves para entender la necesidad de dosis menores y la satisfacción con el tratamiento recibido. El rendimiento cognitivo es precario y se relaciona negativamente con la dosis, lo que puede llevar a importantes dificultades de integración, al repercutir en las actividades de la vida diaria. Sólo aparecen razones que hagan recomendable el uso de dosis altas en aquellos pacientes que persistan en el consumo inyectado de heroína, para disminuir la probabilidad de estos consumos, hasta que se produzca la estabilización. Futuros estudios deberán analizar más detalladamente el papel de cada una de estas variables en los procesos de recuperación y normalización de la vida de estas personas. Se requieren programas de calidad que aborden no sólo la cuestiones farmacológicas ligadas a la adicción, sino también las variables intrapersonales y las condiciones ambientales que pueden favorecer el éxito del programa y la normalización de la vida de los usuarios, o, por el contrario, el incumplimiento terapéutico y el mantenimiento de la conducta adictiva. Los programas de tratamiento con metadona deben orientarse progresivamente a la persona, valorando sus opiniones, fomentando su participación activa en el proceso y mejorando sus niveles de calidad de vida, de modo que el abordaje de su problemática sea similar al que se desarrolla frente a cualquier otro problema de salud.

\section{Conflicto de intereses}

Los autores declaran no presentar conflictos de intereses. Grupo MethaQoL: Madrid: Barreda Marina, M. A., Bartolomé Gil, C., Bosque Coro, S., Callejo Escobar, J., de Ema López, I., Dominguez Aranda, M. A., Ferrero Herreros, Y. E., Galera García, Ó., Garrido Ureña, B., Gil de Bernabe Lopez, M. J., González Galnares, I. R., Gutiérrez Cáceres, S., Heras Dolader, S., Hernández Tejada, C., López Jiménez, M. C., López Zurita, C., Martin Carmona, G., Notario Poves, P., Olmos Espinosa, R., Pacheco Otoya, G., Pérez Carrasco, E., Pérez López, G., Puerta García, C., Rojo Mota, G., Sáez Maldonado, A., Salgado Marcos, N., San Juan Sanz, P., Sújar Plaza, M. I. Extremadura: Borrallo Berjón, M. J., Boticario Villarroel, M. V., Bueno Pozo, R., Iglesias Jiménez, M. F., Mateos Ayucar, M. P.

\section{Referencias}

Adelson, M., Wilson, H. W., Celeste, V. Y., Linzy, S., Kreek, M. J. y Peles, E. (2013). Methadone maintenance treatment experience in Macao. Prospective follow-up for initial 4.5 years. Journal of Psychoactive Drugs, 45, 313-321. doi:10.1080/02791072.2013.825032

Al-Tayyib, A. y Koester, S. (2011). Injection drug users' experience with and attitudes toward methadone clinics in Denver, CO. Journal of Substance Abuse Treatment, 41, 30-36. doi:10.1016/j.jsat.2011.01.009

Amato, L., Davoli, M., Perucci, C. A., Ferri, M., Faggiano, F. y Mattick, R. P. (2005). An overview of systematic reviews of the effectiveness of opiate maintenance therapies: available evidence to inform clinical practice and research. Journal of Substance Abuse Ttreatment, 28, 321-329. doi:10.1016/j.jsat.2005.02.007

Banys, P., Tusel, D. J., Sees, K. L., Reilly, P. M. y Delucchi, K. L. (1994). Low (40 mg) versus high (80 mg) dose methadone in a 180-day heroin detoxification program. Journal of Substance Abuse Treatment, 11, 225-232. doi:10.1016/0740-5472(94)90079-5

Baumeister, M., Vogel, M., Dürsteler-MacFarland, K. M., Gerhard, U., Strasser, J., Walter, M., ... Petitjean, S. A. (2014). Association between methadone dose and concomitant cocaine use in methadone maintenance treatment: a register-based study. Substance Abuse Treatment, Prevention, and Policy, 9, 46. doi:10.1186/1747-597X-9-46

Benyamin, R, Trescot, A. M., Datta, S., Buenaventura, R., Adlaka, R., Sehgal, N., ... Vallejo, R. (2008). Opioid complications and side effects. Pain Physician, 11, S105-S120.

Bell, J. y Zador, D. (2000). A risk-benefit analysis of methadone maintenance treatment. Drug Safety, 22, 179-190. doi:10.2165/00002018-200022030-00002

Bileviciute-Ljungar, I., Häglund, V., Carlsson, J. y von Heijne, A. (2014). Clinical and radiological findings in methadone-induced delayed leukoencephalopathy. Journal of Rehabilitation Medicine, 46, 828-830. doi:10.2340/16501977-1820 
Blaney, T. y Craig, R. J. (1999). Methadone maintenance: does dose determine differences in outcome? Journal of Substance Abuse Treatment, 16, 221-228. doi:10.1016/ S0740-5472(98)00031-2

Bourgois, P. (2000). Disciplining addictions: The bio-politics of methadone and heroin in the United States. Culture, Medicine and Psychiatry, 24, 165-195. doi:10.1023/A:1005574918294

Bracken, B. K., Trksak, G. H., Penetar, D. M., Tartarini W. L., Maywalt, M. A., Dorsey, C. M. y Lukas, S. E. (2012). Response inhibition and psychomotor speed during methadone maintenance: impact of treatment duration, dose, and sleep deprivation. Drug and Alcohol Dependence, 125, 132-139. doi:10.1016/j.drugalcdep.2012.04.004

Brugal, M. T., Domingo-Salvany, A., Puig, R., Barrio, G., García de Olalla, P. y de la Fuente, L. (2005). Evaluating the impact of methadone maintenance programmes on mortality due to overdose and aids in a cohort of heroin users in Spain. Addiction, 100, 981-989. doi:10.1111/ j.1360-0443.2005.01089.x

Bourgois, P. (2000). Disciplining addictions: The bio-politics of methadone and heroin in the United States. Culture, Medicine and Psychiatry, 24, 165-195.

Chen, I. C., Chie W. C., Hwu, H. G., Chou, S. Y., Yeh, Y. C., Yu, C. Y. y Tan, H. K. L. (2011). Alcohol use problem among patients in methadone maintenance treatment in Taiwan. Journal of Substance Abuse Treatment, 40, 142149. doi:10.1016/j.jsat.2010.09.004

Chertkow, H., Nasreddine, Z., Johns, E., Phillips, N. y McHenry, C. (2011). The Montreal Cognitive Assessment (MoCA): Validation of alternate forms and new recommendations for education corrections. Alzheimer's E D Dmentia, 7, S157. doi:10.1016/j.jalz.2011.05.423

Chugh, S. S., Socoteanu, C., Reinier, K., Waltz, J., Jui, J. y Gunson, K. (2008). A community-based evaluation of sudden death associated with therapeutic levels of methadone. American Journal of Medicine, 121, 66-71. doi:10.1016/j.amjmed.2007.10.009

Cohen,J. (1973).Eta-squared and partial eta-squared in fixed factor ANOVA designs. Educational and Psychological Measurement, 33, 107-112. doi:10.1177/001316447303300111

Cohen, J. (1988). Statistical power analysis for the behavioral sciences $\left(2^{a}\right.$ ed.). Hillsdale, NJ: Erlbaum.

Copersino, M. L., Schretlen, D. J., Fitzmaurice, G., Lukas, S. E., Faberman, J., Sokoloff, J. y Weiss, R. D. (2012). Effects of cognitive impairment on substance abuse treatment attendance: predictive validation of a brief cognitive screening measure. American Journal of Drug and Alcohol Abuse, 38, 246-250. doi:10.3109/00952990.2 012.670866

Curran, H. V., Bolton, J., Wanigaratne, S. y Smyth, C. (1999). Additional methadone increases craving for heroin: a double-blind, placebocontrolled study of chronic opiate users receiving methadone substitution treatment. Addiction, 94, 665-674. doi:10.1046/j.13600443.1999.9456654.x

Darke, S., McDonald, S., Kaye, S. y Torok, M. (2012). Comparative patterns of cognitive performance amongst opioid maintenance patients, abstinent opioid users and non-opioid users. Drug and Alcohol Dependence, 126, 309-315. doi:10.1016/j.drugalcdep.2012.05.032

D’Aunno, T., Folz-Murphy, N. y Lin, X. (1999). Changes in methadone treatment practices: results from a panel study, 1988-1995. American Journal of Drug and Alcohol Abuse, 25, 681-699. doi:10.1081/ADA-100101886

D’Aunno, T., Pollack, H. A., Frimpong, J. A. y Wuchiett, D. (2014). Evidence-based treatment for opioid disorders: A 23-year national study of methadone dose levels. Journal of Substance Abuse Treatment, 47, 245-250. doi:10.1016/j.jsat.2014.06.001

Deering, D., Sheridan, J., Sellman, J., Adamson, S., Pooley, S., Robertson, R.y Henderson, C. (2011). Consumer and treatment provider perspectives on reducing barriers to opioid substitution treatment and improving treatment attractiveness. Addictive Behaviors, 36, 636-642. doi:10.1016/j.addbeh.2011.01.004

De Las Cuevas, C., Gonzalez de Rivera, J. L., Henry Benitez, M., Monterrey, A. L., Rodriguez-Pulido, F. y Gracia Marco, R. (1991). Análisis factorial de la versión española del SCL-90-R en la población general. Anales de Psiquiatria, 7, 93-96.

De Maeyer, J., Vanderplasschen, W., Camfield, L., Vanheule, S., Sabbe, B. y Broekaert, E. (2011). A good quality of life under the influence of methadone: A qualitative study among opiate-dependent individuals. International Journal of Nursing Studies, 48, 1244-1257. doi:10.1016/j. ijnurstu.2011.03.009

De Maeyer, J., Vanderplasschen, W., Lammertyn, J., van Nieuwenhuizen, C., Sabbe, B. y Broekaert, E. (2011). Current quality of life and its determinants among opiate-dependent individuals five years after starting methadone treatment. Quality of Life Research, 20, 139-150. doi:10.1007/s11136-010-9732-3

Derogatis, L. R. (1992). SCL-90-R: Administration, scoring and procedures manual for the $R$ (evised) version and other instruments of the psychopathology rating scale series. Towson: Clinical Psychometric Research.

Dobler-Mikola, A., Hattenschwiler, J., Meili, D., Beck, T., Boni, E., Modestin, J. (2005). Patterns of heroin, cocaine, and alcohol abuse during long-term methadone maintenance treatment. Journal of Substance Abuse Treatment, 29, 259-265. doi :10.1016/j.jsat.2005.08.002

Eiden, C., Leglis, Y., Clarivet, B., Blayac, J. P. y Peyrière, H. (2012). Co-morbidités psychiatriques associées à des fortes posologies de méthadone $(>100 \mathrm{mg} / \mathrm{j})$ : analyse rétrospective d'une cohorte de patients traités. Thérapie, 67, 223-230. doi :10.2515/therapie/2012025 
Faggiano, F., Vigna-Taglianti, F., Versino, E. y Lemma, P. (2003). Methadone maintenance at different dosages for opioid dependence. Cochrane Database of Systematic Reviews, 3, CD002208. doi:10.1002/14651858.CD002208

Fareed, A., Vayalapalli, S., Stout, S., Casarella, J., Drexler, K. y Bailey, S. P. (2010). Effect of methadone maintenance treatment on heroin craving, a literature review. Journal of Addictive Diseases, 30, 27-38. doi:10.1080/10550887.2010.531672

Farré, M., Mas, A., Torrens, M., Moreno, V. y Camí, J. (2002). Retention rate and illicit opioid use during methadone maintenance interventions: a meta-analysis. Drug and Alcohol Dependence, 65, 283-290. doi:10.1016/ S0376-8716(01)00171-5

Fernández Miranda, J. J. (2001). Efectividad de los programas de mantenimiento con metadona. Una revisión de los resultados de los estudios de evaluación. Medicina Clínica (Barcelona), 116, 150-154.

Fernández Miranda, J. J. (2004). Calidad asistencial y cronicidad en los programas de mantenimiento con agonistas opiáceos. Adicciones, 16, 109-116.

Fernández Miranda, J. J., González García-Portilla, M. P., Sáiz Martínez, P.A., Gutiérrez Cienfuegos, E, Bobes García, J. (1999). Calidad de vida y severidad de la adicción en heroinómanos en mantenimiento prolongado con metadona. Adicciones, 11, 43-52.

González de Rivera, J. L., Derogatis, L. R., de las Cuevas, C., Gracia Marco, R., Rodríguez-Pulido, F., Henry-Benítez, M. y Monterrey, A. L. (1989). The Spanish version of the SCL-90-R. Normative data in the general population. Towson: Clinical Psychometric Research.

Grönbladh, L. y Öhlund, L. S. (2011). Self-reported differences in side-effects for 110 heroin addicts during opioid addiction and during methadone treatment. $\mathrm{He}$ roin Addiction and Related Clinical Problems, 13, 5-12.

Gruber, S. A., Tzilos, G. K., Silveri, M. M., Pollack, M., Renshaw, P. F., Kaufman, M. J. y Yurgelun-Todd, D. A. (2006). Methadone maintenance improves cognitive performance after two months of treatment. Experimental and Clinical Psychopharmacology, 14, 157-164. doi:10.1037/1064-1297.14.2.157

Hall, W., Carter, A. y Forlini, C. (2015). The brain disease model of addiction: is it supported by the evidence and has it delivered on its promises? Lancet Psychiatry, 2, 105110. doi:10.1016/S2215-0366(14)00126-6

Hammer, R., Dingel, M., Ostergren, J., Partridge, B., McCormick, J. y Koenig, B. A. (2013). Addiction: Current criticism of the brain disease paradigm. American Journal of Bioethics Neuroscience, 4, 27-32. doi:10.1080/21507740 .2013 .796328

Habrat, B., Chmielewska, K., Baran-Furga, H., Keszycka, B. y Taracha, E. (2002). Subjective Quality of Life in opiate-dependent patients before admission after six months and one-year participation in methadone program. Przegl Lek, 59, 351-354.
Harris, J. y McElrath, K. (2012). Methadone as social control: Institutionalized stigma and the prospect of recovery. Qualitative Health Research, 22, 810-824. doi:10.1177/1049732311432718

Harris, M. y Rhodes, T. (2013). Methadone diversion as a protective strategy: the harm reduction potential of 'generous constraints'. International Journal of Drug Policy, 24, e43-e50. doi:10.1016/j.drugpo.2012.10.003

Hartgers, C., van den Hoek, A., Krijnen, P. y Coutinho, R. A. (1992). HIV prevalence and risk behavior among injecting drug users who participate in 'Low-Threshold' methadone programs in Amsterdam. American Journal of Public Health, 82, 547-551. doi:10.2105/AJPH.82.4.547

He, Q., Wang, X., Xia, Y., Mandel, J. S., Chen, A., Zhao, L., ... Ling, L. (2011). New community-based methadone maintenance treatment programs in Guangdong, China, and their impact on patient quality of life. Substance Use E्F Misuse, 46, 749-757. doi:10.3109/10826084.2010. 534124

Hooper, D., Coughlan, J. y Mullen, M. R. (2008). Structural equation modelling: Guidelines for determining model fit. Electronic Journal of Business Research Methods, 6, 53- 60.

Karow, A., Verthein, U., Pukrop, R., Reimer, J., Haasen, C., Krausz, M. y Schäfer, I. (2011). Quality of life profiles and changes in the course of maintenance treatment among 1,015 patients with severe opioid dependence. Substance Use Ẽ Misuse, 46, 705-715. doi:10.3109/10826 084.2010 .509854

Katz, N. (2005). The impact of opioids on the endocrine system. Pain Management Rounds, 1, 1-6. doi:10.1097/ AJP.0b013e3181850df6

Keen, J., Oliver, P., Rowse, G. y Mathers, N. (2003). Does methadone maintenance treatment based on the new national guidelines work in a primary care setting? British Journal of General Practice, 53, 461-467.

Kelly, S. M., O'Grady, K. E., Brown, B. S., Mitchell, S. G. y Schwartz, R. P. (2010). The role of patient satisfaction in methadone treatment. American Journal of Drug and Alcohol Abuse, 36, 150-154. doi:10.3109/00952991003736371

Kelly, S. M., O’Grady, K. E., Brown, B. S., Mitchell, S. G. y Schwartz, R. P. (2011). Predictors of methadone treatment retention from a multi-site study: A survival analysis. Drug $\mathcal{E}$ Alcohol Dependence, 117, 170-175. doi:10.1016/j.drugalcdep.2011.01.008

Krantz, M. J., Kutinsky, I. B., Robertson, A. D. y Mehler, P. S. (2003). Dose-related effects of methadone on QT prolongation in a series of patients with torsade de pointes. Pharmacotherapy, 23, 802-805. doi:10.1592/ phco.23.6.802.32186

Krantz, M. J., Martin, J., Stimmel, B., Mehta, D. y Haigney, M. C. P. (2009). QTc interval screening in methadone treatment. Annals of Internal Medicine, 150, 387-395. doi:10.7326/0003-4819-150-1-200903170-00104 
Langendam, M. W., Van Brussel, G. H., Coutinho, R. A. y Van Ameijden, E. J. (2001). The impact of harm-reduction-based methadone treatment on mortality among heroin users. American Journal of Public Health, 91, 774780. doi:10.2105/AJPH.91.5.774

Leavitt, S. B. (2003). Methadone dosing \& safety in the treatment of opioid addiction. Addiction Treatment Forum, 12, 1-8.

Leshner, A. I. (1997). Addiction is a brain disease, and it matters. Science, 278, 45-47. doi:10.1126/science.278.5335.45

Liao, D. L., Chen, P. C., Chen, C. H., Hsieh, C. J., Huang, Y. F., Shih, W. Y. y Cheng, J. J. (2013). Higher methadone doses are associated with lower mortality in patients of opioid dependence in Taiwan. Journal of Psychiatric Research, 47, 1530-1534. doi:10.1016/j.jpsychires.2013.07.001

Lina, C., Wu, Z. y Detels, R. (2011). Family support, quality of life and concurrent substance use among methadone maintenance therapy clients in China. Public Health, 125, 269-274. doi:10.1016/j.puhe.2011.01.009

Lingford-Hughes, A. R., Welch, S. y Nutt, D. J. (2004). Evidence-based guidelines for the pharmacological management of substance misuse, addiction and comorbidity: recommendations from the British Association for Psychopharmacology. Journal of Psychopharmacology, 18, 293-336. doi:10.1177/0269881104048516

Liu, E., Rou, K., McGoogan, J. M., Pang, L., Cao, X., Wang, C., ... y Wu, Z. (2013). Factors associated with mortality of HIV-positive clients receiving methadone maintenance treatment in China. Journal of Infectious Diseases, 208, 442-453. doi:10.1093/infdis/jit163

Loeber, S., Kniest, A., Diehl, A., Mann, K. y Croissant, B. (2008). Neuropsychological functioning of opiate-dependent patients: A nonrandomized comparison of patients preferring either buprenorphine or methadone maintenance treatment. American Journal of Drug and Alcohol Abuse, 34, 584-593. doi:10.1080/00952990802308239

Lucas-Carrasco, R. (2012). The WHO quality of life (WHOQOL) questionnaire: Spanish development and validation studies. Quality of Life Research, 21, 161-165. doi:10.1007/s11136-011-9926-3

Maddux, J. F., Desmond, D. P. y Vogtsberger, K. N. (1995). Patient-regulated methadone dose and optional counseling in methadone maintenance. American Journal on Addictions, 4, 18-32. doi:10.1111/j.1521-0391.1995.tb00255.x

Maddux, J. F., Prihoda, T. J. y Vogtsberger, K. N. (1997). The relationship of methadone dose and other variables to outcomes of methadone maintenance. American Journal on Addictions, 6, 246-255. doi:10.1111/j.1521-0391.1997. tb00404.x

Maremmani, I., Pacini, M., Lubrano, S. y Lovrecic, M. (2003). When "enough" is still not "enough": effectiveness of high-dose methadone in the treatment of heroin addiction. Heroin Addiction E Related Clinical Problems, 5, 17-32.

Mattick, R. P., Breen, C., Kimber, J. y Davoli, M. (2009). Methadone maintenance therapy versus no opioid replacement therapy for opioid dependence. Cochrane Database of Systematic Reviews, 3, CD002209. doi:10.1002/14651858.CD002209.pub2

Maxwell, S. y Shinderman, M. (1999). Optimizing response to methadone maintenance treatment: use of higher-dose methadone. Journal of Psychoactive Drugs, 31, 95-102. doi:10.1080/02791072.1999.10471730

Millson, P., Challacombe, L., Villeneuve, P. J., Strike, C. J., Fischer, B., Myers, T., ... Hopkins, S. (2006). Determinants of health-related quality of life of opiate users at entry to low-threshold methadone programs. European Addiction Research, 12, 74-82. doi:10.1159/000090426

Millson, P. E., Challacombe, L., Villeneuve, P. J., Fischer, B., Strike, C. J., Myers, T., ... Pearson, M. (2004). Self-perceived health among Canadian opiate users: a comparison to Canadian the general population and to other chronic disease populations. Canadian Journal of Public Health, 95, 99-103.

Millson, P., Challacombe, L., Villeneuve, P. J., Strike, C. J., Fischer, B., Myers, T., ... Hopkins, S. (2007). Reduction in injection-related hiv risk after 6 months in a low-threshold methadone treatment program. AIDS Education and Prevention, 19, 124-136. doi:10.1521/ aeap.2007.19.2.124

Mino, A., Page, D., Dumont, P. y Broers, B. (1998). Treatment failure and methadone dose in a public methadone maintenance treatment programme in Geneva. Drug and Alcohol Dependence, 50, 233-239. doi:10.1016/S03768716(98)00035-0

Mintzer, M. Z., Copersino, M. L. y Stitzer, M. L. (2005). Opioid abuse and cognitive performance. Drug and Alcohol Dependence, 78, 225-230. doi:10.1016/j.drugalcdep.2004.10.008

Mintzer, M. Z. y Stitzer, M. L. (2002) . Cognitive impairment in methadone maintenance patients. Drug and Alcohol Dependence, 67, 41-51. doi:10.1016/S0376-8716(02)00013-3

Montgomery, L. T., Sanning, B., Litvak, N. y Peters, E. N. (2014). Preliminary findings on the association between clients' perceived helpfulness of substance abuse treatment and outcomes: Does race matter? Drug and Alcohol Dependence, 139, 152-158. doi:10.1016/j.drugalcdep.2014.03.026

Musshoff, F., Trafkowski, J., Lichtermann, D. y Madea, B. (2010). Comparison of urine results concerning co-consumption of illicit heroin and other drugs in heroin and methadone maintenance programs. International Journal of Legal Medicine, 124, 499-503. doi:10.1007/s00414-0090361-8

Nasreddine, Z. S., Phillips, N. A., Bédirian, V., Charbonneau, S., Whitehead, V., Collin, I.... Chertkow, H. 
(2005). The Montreal Cognitive Assessment, MoCA: a brief screening tool for mild cognitive impairment. Journal of the American Geriatrics Society, 53, 695-699. doi:10.1111/j.1532-5415.2005.53221.x

Nosyk, B., Guh, D. P., Sun, H., Oviedo-Joekes, E., Brissette, S., Marshd, D. C., ... Anis, A. H. (2011). Health related quality of life trajectories of patients in opioid substitution treatment. Drug and Alcohol Dependence, 118, 259264. doi:10.1016/j.drugalcdep.2011.04.003

Nosyk, B., Marsh, D. C., Sun, H., Schechter, M. T. y Anis, A. H. (2010). Trends in methadone maintenance treatment participation, retention, and compliance to dosing guidelines in British Columbia, Canada: 1996-2006. Journal of Substance Abuse Treatment, 39, 22-31. doi:10.1016/j. jsat.2010.03.008

Notley, C., Blyth, A., Maskrey, V., Pinto, H. y Holland, R. (2015). Exploring the concepts of abstinence and recovery through the experiences of long-term opiate substitution clients. Substance Abuse, 36, 232-239. doi:10.1080/ 08897077.2014 .941085

Pacini, M., Maremmani, A. G. I., Rovai, L., Rugani, F. y Maremmani, I. (2010). Treating heroin addicts. Blocking dosages and stimulation-stabilization of opioidergic system. Heroin Addiction E Related Clinical Problems, 12, 41-48.

Padaiga, Z., Subata, E. y Vanagas. G. (2007). Outpatient methadone maintenance treatment program. Quality of life and health of opioid-dependent persons in Lithuania. Medicina (Kaunas), 4, 235-241.

Pedrero Pérez, E. J. y López-Durán, A. (2005). Autoinformes de sintomatología depresiva en drogodependientes: nivel de coincidencia del BDI, SCL-90-R y MCMI-II. ¿Depresión o malestar inespecífico? Adicciones, 17, 215-230.

Pérez de los Cobos, J., Fidel, G., Escuder, G., Haro, G., Sánchez, N., Pascual, C.,... , Trujols, J. (2004). A satisfaction survey of opioid-dependent clients at methadone treatment centres in Spain. Drug and Alcohol Dependence, 73, 307-313. doi:10.1016/j.drugalcdep.2003.11.001

Perreault, M., Héroux, M. C., White, N. D., Lauzon, P., Mercier, C. y Rousseau, M. (2007). Treatment retention and evolution of clientele in a low threshold methadone substitution treatment program in Montreal. Canadian Journal of Public Health, 98, 33-36.

Peterson, J. A., Schwartz, R. P., Mitchell, S. G., Reisinger, H. S., Kelly, S. M., O'Grady, K. E.... Agar, M. H.. (2010). Why don't out-of-treatment individuals enter methadone treatment programmes? International Journal of Drug Policy, 21, 36-42. doi:10.1016/j.drugpo.2008.07.004

Preston, K. L., Umbricht, A. y Epstein, D. H. (2000). Methadone dose increase and abstinence reinforcement for treatment of continued heroin use during methadone maintenance. Archives of General Psychiatry, 57, 395404. doi:10.1001/archpsyc.57.4.395.

Rass, O., Kleykamp, B. A., Vandrey, R. G., Bigelow, G. E., Leoutsakos, J. M., Stitzer, M. L., ... Mintzer, M. Z. (2014).
Cognitive performance in methadone maintenance patients: effects of time relative to dosing and maintenance dose level. Experimental and Clinical Psychopharmacology, 22, 248-256. doi:10.1037/a0035712

Rodríguez, M., Jiménez-Lerma, J. M., Iraurgi, I., Murua, F., Bacigalupe, L., Chavarri, M. R., \& Balsategi, B. (2002). Evaluación de la satisfacción con el tratamiento en un centro ambulatorio de drogodependencias a través del Treatment Perceptions Questionnaire (TPQ). Adicciones, 14, 1-8.

Rojo-Mota, G., Pedrero-Pérez, E. J., Ruiz-Sánchez de León, J. M., Llanero-Luque, M. y Puerta-García, C. (2013). Cribado neurocognitivo en adictos a sustancias: la evaluación cognitiva de Montreal. Revista de Neurología, 56, 129-136.

Roncero, C., Fuste, G., Barral, C., Rodríguez-Cintas, L, Martínez-Luna, N., Eiroa-Orosa, F. J., Casas, M, on behalf of the PROTEUS study investigators. (2011). Therapeutic management and comorbidities in opiate-dependent patients undergoing a replacement therapy programme in Spain: the PROTEUS study. Heroin Addiction and Related Clinical Problems, 13, 5-16.

Roy, A. K., McCarthy, C., Kiernan, G., McGorrian, C., Keenan, E., Mahon, N. G. y Sweeney, B. (2012). Increased incidence of QT interval prolongation in a population receiving lower doses of methadone maintenance therapy. Addiction, 107, 1132-1139. doi:10.1111/j.13600443.2011.03767.x

Ruggeri, M., Lasalvia, A., Dall'Agnola, R., Van Wijngaarden, B., Knudsen, H.C., Leese, M., .... y The Epsilon Study Group (2000). Development, internal consistency and reliability of the Verona Service Satisfaction Scale - European Version. British Journal of Psychiatry, 177, s41-s48. doi:10.1192/bjp.177.39.s41

Schwartz, R. P., Highfield, D. A., Jaffe, J. H., Brady, J. V., Butler, C. B., Rouse, C. O., ... Breteler, M. M. B. (2006). A randomized controlled trial of interim methadone maintenance. Archives of General Psychiatry, 63, 102-109. doi:10.1001/archpsyc.63.1.102.

Senbanjo, R., Wolff, K. I. M., Marshall, E y Strang, J. (2009). Persistence of heroin use despite methadone treatment: Poor coping self-efficacy predicts continued heroin use. Drug and Alcohol Review, 28, 608-615. doi:10.1111/j.14653362.2009.00064.x

Servicio Andaluz de Salud (2010). Desarrollo de Programas de Tratamiento Asertivo Comunitario en Andalucía. Documento marco. Anexo 3.1. Granada: Área de Dirección de Organizaciones Sanitarias de la Escuela Andaluza de Salud Pública.

Skevington, S. M., Lotfy, M. y O'Connell, K. A. (2004). The World Health Organization's WHOQOL-BREF quality of life assessment: psychometric properties and results of the international field trial. A report from the WHOQOL group. Quality of life Research, 13, 299-310. doi:10.1023/B:QURE.0000018486.91360.00 
Skevington, S. M., Sartorius, N., Amir, M., and THE WHOQOL Group. (2004). Developing methods for assessing quality of life in different cultural settings. Social Psychiatry and Psychiatric Epidemiology, 39, 1-8. doi:10.1007/ s00127-004-0700-5

Torrens, M., Castillo, C. y Perez-Sola, V. (1996). Retention in a low threshold methadone maintenance program. Drug and Alcohol Dependence, 41, 55-59. doi:10.1016/03768716(96)01230-6

Torrens, M., Domingo-Salvany, A., Alonso, J., Castillo, C. y San, L. (1999). Methadone and quality of life. Lancet, 353, 1101. doi:10.1016/S0140-6736(05)76462-X

Trujols, J., Portella, M. J., Iraurgi, I., Campins, M. J., Siñol, N. y Pérez de los Cobos, J. (2013). Patient-reported outcome measures: Are they patient-generated, patient-centred or patient-valued? Journal of Mental Health, 22, 555-562. doi:10.3109/09638237.2012.734653

Trujols, J. y Pérez de los Cobos, J. (2005). La perspectiva de los usuarios sobre los tratamientos de mantenimiento con metadona: una revisión centrada en la satisfacción con el tratamiento. Adicciones, 17, s181-s204.

Van Ameijden, E. J., Langendam, M. W. y Coutinho, R. A. (1999). Dose-effect relationship between overdose mortality and prescribed methadone dosage in low threshold maintenance programs. Addictive Behaviours, 24, 559-563. doi:10.1016/S0306-4603(98)00083-5

Vanderplasschen, W., Naert, J., Vander Laenen, F. y De Maeyer, J. (2015). Treatment satisfaction and quality of support in outpatient substitution treatment: opiate users' experiences and perspectives. Drugs: education, prevention, and policy, 22, 272-280. doi:10.3109/0968763 7.2014 .981508

Verdejo, A., Toribio, I., Orozco, C., Puente, K. L. y Pérez-García, M. (2005). Neuropsychological functioning in methadone maintenance patients versus abstinent heroin abusers. Drug and Alcohol Dependence, 78, 283-288. doi:10.1016/j.drugalcdep.2004.11.006

Villeneuve, P. J., Challacombe, L., Strike, C. J., Myers, T., Fischer, B., Shore, R., Hopkins, S. y Millson, P. E. (2006). Change in health-related quality of life of opiate users in low-threshold methadone programs. Journal of Substance Use, 11, 137-149. doi:10.1080/14659890500256945

Walker, P. W., Klein, D. y Kasza, L. (2003). High dose methadone and ventricular arrhythmias: a report of three cases. Pain, 103, 321-324. doi:10.1016/S03043959(02)00461-X

Wang, P. W., Wu, H. C., Yen, C. N., Yeh, Y. C., Chung, K. S., Chang, H. C. y Yen C, F. (2012). Change in quality of life and its predictors in heroin users receiving methadone maintenance treatment in Taiwan: An 18-month follow-up study. American Journal of Drug and Alcohol Abuse, 38, 213-219. doi:10.3109/00952990.2011.649222

Webster, L. R. (2013). Methadone side effects: Constipation, respiratory depression, sedation, sleep-disordered breathing, and the endocrine system. En Cruciani, R. A. y Knotkova H., Handbook of Methadone Prescribing and Buprenorphine Therapy (pp. 39-49). New York: Springer.

White, W. y Mojer-Torres, L. (2010). Recovery-oriented methadone maintenance. Chicago (IL): Great Lakes Addiction Technology Transfer Center, Philadelphia Department of Behavioral Health and Mental Retardation Services and Northeast Addiction Technology Transfer Center.

WHO (2004). The World Health Organization Quality of Life (WHOQOL)-BREF World Health Organization. Recuperado de http://www.who.int/substance_abuse/research_ tools/whoqolbref/en/

Zeldman, A., Ryan, R. M. y Fiscella, K. (2004). Motivation, autonomy support, and entity beliefs: Their role in methadone maintenance treatment. Journal of Social and Clinical Psychology, 23, 675-696. doi:10.1521/ jscp.23.5.675.50744

\section{Anexo I. Escala de Satisfacción utilizada}

1. ¿Cuál es su impresión general sobre la eficacia del CAD para ayudarle a afrontar sus problemas?

2. ¿Cuál es su impresión general sobre la capacidad para escucharle y comprender sus problemas de los profesionales del CAD?

3. ¿Cuál es su impresión general sobre el comportamiento y el trato personal recibido por parte de los profesionales del CAD?

4. ¿Cuál es su impresión general sobre la capacidad de los profesionales del CAD para colaborar, cuando es necesario, con su médico de familia u otros especialistas?

5. ¿Cuál es su impresión general sobre todos los servicios que usted ha recibido en el CAD?

6. ¿Cuál es su impresión general sobre la eficacia del centro para ayudarle a mejorar la relación con sus familiares más cercanos?

7. ¿Cuál es su impresión general sobre la eficacia del centro para ayudar a sus familiares más cercanos a conocer y comprender mejor los problemas de usted?

8. ¿Cuál es su impresión general sobre el conocimiento de los profesionales del CAD de sus enfermedades actuales y pasadas?

9. ¿Cuál es su impresión general sobre la información que recibió sobre el diagnóstico y la posible evolución de su adicción?

10. ¿Cuál es su impresión general sobre la eficacia del centro en ayudarle a mejorar sus relaciones con personas fuera de su ambiente familiar (amigos, vecinos, compañeros de trabajo)?

11. ¿Cuál es su impresión general sobre la claridad y concreción de las instrucciones recibidas sobre lo que tenía que hacer entre consulta y consulta?

12. ¿Cuál es su impresión general sobre la eficacia del centro para ayudarle a mejorar su capacidad de cuidarse a sí mismo (por ejemplo, la higiene personal, su alimentación, su alojamiento, etc.)?

13. ¿Cuál es su impresión general sobre la ayuda que usted ha recibido en el caso de presentar efectos secundarios y molestos de los medicamentos?

Opciones de respuesta: 1 Malísima; 2 Generalmente insatisfactoria; 3 Ni buena ni mala; 4 Generalmente satisfactoria; 5 Excelente 